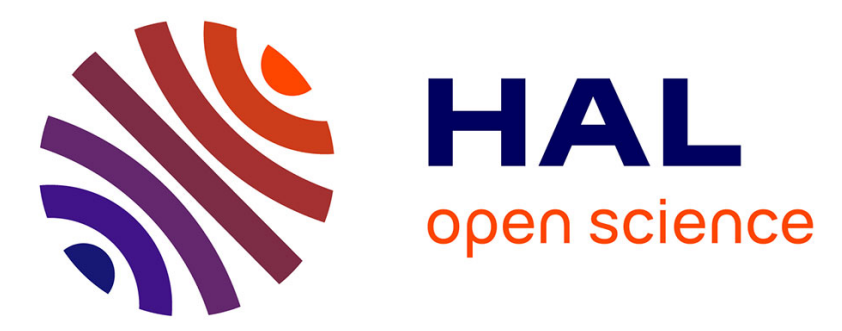

\title{
Percolation in three-dimensional fracture networks for arbitrary size and shape distributions
}

\author{
J.-F. Thovert, Valeri V. Mourzenko, P. M Adler
}

\section{To cite this version:}

J.-F. Thovert, Valeri V. Mourzenko, P. M Adler. Percolation in three-dimensional fracture networks for arbitrary size and shape distributions. Physical Review E , 2017, 95 (4), 10.1103/PhysRevE.95.042112 . hal-03106028v2

\section{HAL Id: hal-03106028 \\ https://hal.science/hal-03106028v2}

Submitted on 28 Jan 2021

HAL is a multi-disciplinary open access archive for the deposit and dissemination of scientific research documents, whether they are published or not. The documents may come from teaching and research institutions in France or abroad, or from public or private research centers.
L'archive ouverte pluridisciplinaire HAL, est destinée au dépôt et à la diffusion de documents scientifiques de niveau recherche, publiés ou non, émanant des établissements d'enseignement et de recherche français ou étrangers, des laboratoires publics ou privés. 


\title{
Percolation in three-dimensional fracture networks for arbitrary size and shape distributions
}

\author{
J.-F. Thovert" and V. V. Mourzenko ${ }^{\dagger}$ \\ Institut Pprime - CNRS, SP2MI, BP 30179, 86962 Futuroscope Chasseneuil Cedex, France \\ P. M. Adler \\ UPMC METIS. Boîte 105, 4 place Jussieu, 75252 Paris Cedex 05, France \\ (Received 29 November 2016; published 7 April 2017)
}

\begin{abstract}
The percolation threshold of fracture networks is investigated by extensive direct numerical simulations. The fractures are randomly located and oriented in three-dimensional space. A very wide range of regular, irregular, and random fracture shapes is considered, in monodisperse or polydisperse networks containing fractures with different shapes and/or sizes. The results are rationalized in terms of a dimensionless density. A simple model involving a new shape factor is proposed, which accounts very efficiently for the influence of the fracture shape. It applies with very good accuracy in monodisperse or moderately polydisperse networks, and provides a good first estimation in other situations. A polydispersity index is shown to control the need for a correction, and the corrective term is modelled for the investigated size distributions.
\end{abstract}

DOI: 10.1103/PhysRevE.95.042112

\section{INTRODUCTION}

Geological fractures, usually defined as surface discontinuities in rocks, are ubiquitous underground from scales of a few millimeters to hundreds of kilometers [1]. Fluids can generally flow through them and possibly much faster than in the embedding porous matrix. Therefore, fracture networks do influence flow and transports, and they are actively studied in many areas such as geothermal energy exploitation, hydrology, oil, and gas recovery and nuclear waste storage. In particular, it is important to know their statistical geometrical properties. A prominent feature in this respect is their connectivity, and possibly their percolating character, which was first studied by Charlaix et al. [2] and Balberg [3].

This paper intends to provide a major update of some of our earlier works. Monodisperse networks of fractures were considered in [4], where our first unification of the percolation threshold values for various regular polygonal fracture shapes was achieved, by use of the concept of excluded volume. Polydisperse networks with power law distributions of the fracture sizes were addressed in [5], where a generalized dimensionless network density was introduced which accounts for most of the influence of polydispersity. In all these works, and in the present one as well, the fracture network is considered as a given structure and its formation is not addressed.

The present work proceeds along the same lines, with significant improvements and extensions of several kinds. A very extensive set of calculations was conducted, taking benefit of much increased computational capabilities, with a significant precision improvement resulting mostly from the use of much larger and numerous samples. The range of explored situations was also greatly extended, including new fracture shapes very far from circularity, and various kinds

\footnotetext{
*thovert@ensma.fr

†murzenko@ensma.fr

${ }^{\ddagger}$ pierre.adler@upmc.fr
}

of polydispersity, such as mixtures of fractures with different shapes, possibly random, and various size distributions.

The rationalization of the results benefits greatly from these gains in precision and scope of the data. The corrective factor for fractures with very elongated shapes proposed in [5] was successful only for the cases examined by then. A much better model, more accurate and applicable in a much wider range of shapes and mixtures of shapes, is formulated, based on a different shape factor. Effects of the size polydispersity undetected in [5] could be quantified and modelled, and a simple criterion is obtained to assess whether they are a priori negligible or should be taken into account.

The paper is organized as follows. General information is provided in Sec. II, including the description of the networks, dimensionless parameters and concepts used in the analysis, and the numerical aspects of the determination of the percolation thresholds. Monodisperse networks are addressed in Sec. III. More complex networks containing mixtures of fractures of various kinds are explored in Sec. IV and size polydispersity is considered in Sec. V. A heuristic argument proposed in [6] to predict the percolation thresholds in continuum systems is revisited in Sec. VI. Concluding remarks are formulated in Sec. VII.

Additional information is provided in the Supplemental Material [7], including the comprehensive set of numerical data, details about the random quadrilaterals used in some of the investigated networks, and auxiliary data pertaining to Sec. VI.

\section{METHODS}

\section{A. Fracture networks}

A fracture network is defined as a set of individual fractures which possibly intersect. The fractures are regarded as plane, finite objects without any restriction for their shape except for the convexity of their contour.

We consider a canonical situation where several important hypotheses are made. First, the fractures are randomly located in space, with a density $\rho$ (mean number of fractures per 

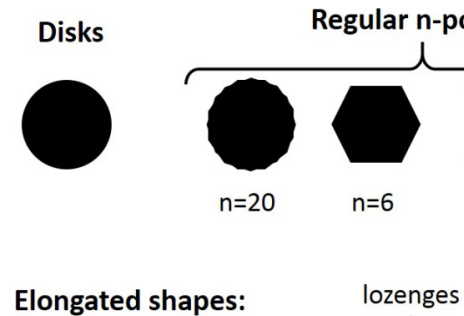

lozenges

Elongated shapes:

(aspect ratio $f=1$ to 16 ) $n=6$

egular n-polygons

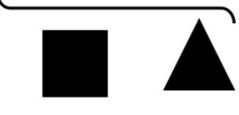

$\mathrm{n}=4$

$n=3$

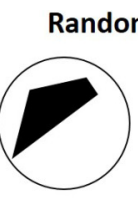

rectangles
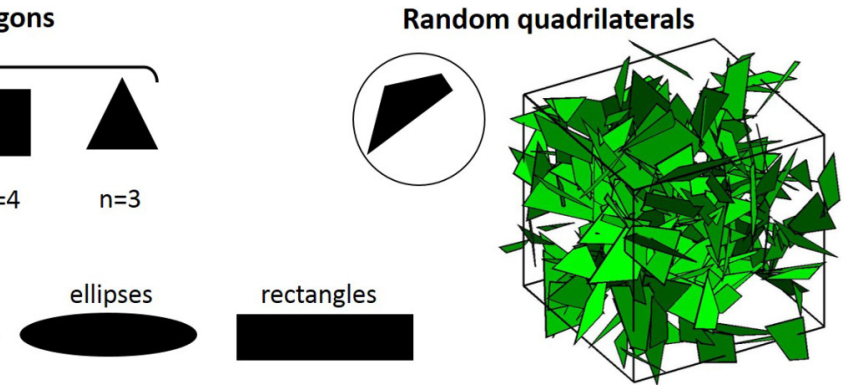

FIG. 1. Illustration of the investigated fracture shapes, and example of a network containing 238 random quadrilaterals.

unit volume). More precisely, the number of fractures in a volume $V$ obeys a Poisson distribution with mean $\rho V$, independent of position. Second, the fractures are randomly and isotropically oriented. Finally, the various attributes of a fracture (position, orientation, shape, size) are uncorrelated, as well as the attributes of different fractures in the network.

These are strong assumptions which are generally not met in real fracture networks. However, earlier works have shown that the percolation properties of anisotropic networks where the fracture orientations are arranged in several discrete families [8] or according to a continuous Fisher distribution around a preferential direction [9] do not strongly differ from those of isotropic networks. It has also been shown [10] that the results for uniform $\rho$ are applicable locally in inhomogeneous networks if the density variations take place over distances larger than the typical fracture size. Therefore, the present canonical situation is a good starting point for the study of more general cases.

The networks are generated by inserting fractures with random orientations and with their centers at random locations and in a cubic cell of size $L^{3}$. Periodicity is applied along the three $x, y$, and $z$ axes.

A wide range of fracture shapes has been considered, including disks and ellipses with an aspect ratio $f=1-16$ (denoted $f$-ellipses), regular polygons with $n=3,4,6$, and 20 vertices, and elongated polygons with aspect ratios $f=1-16$ (see Fig. 1). Note that the $f$-lozenges, $f$-ellipses, and $f$-rectangles belong to the class of the Lamé curves, or superellipses [11],

$$
\left|\frac{x}{a}\right|^{q}+\left|\frac{y}{b}\right|^{q}=1,
$$

with $q=1,2$, and $\infty$, respectively, and $a / b=f$. Mixtures of fractures with different shapes in varying proportions have also been considered.

The case when all the fractures in the network have identical shapes and sizes is denoted $\mathrm{I}^{2}$ OUD in [12] (acronym for identical, isotropically oriented, and uniformly distributed). However, mixtures of fractures with different shapes in varying proportions have also been considered here, and three kinds of fracture size distributions have been investigated: monodisperse, bidisperse with two sizes $R_{M}$ and $R_{m}$ (the fraction of the largest ones is denoted by $\varphi_{M}$ ), and power law distributions. The bidisperse distribution is a simple model without claim for realism; it corresponds to an extreme case which is useful to check our predictions. Power law distribution has a different status since it is one of the most commonly observed distributions in geological fracture networks (see the review in Sec. 5.2.2 of [13]); it is described by

$$
\varphi(R)=\alpha R^{-a} \quad\left(R_{m} \leqslant R \leqslant R_{M}\right)
$$

where $\varphi(R) \mathrm{d} R$ is the probability of $R$ being in the interval $[R, R+d R] ; \alpha$ results from the normalization condition that the integral over $R$ of (2) should be equal to 1 . The exponent $a$ is found to range between 1 and 5 in many observations of fractured rocks [13]. The ratio $R_{M} / R_{m}$ is denoted $\tilde{R}$ in the following, for both bidisperse and power law distributions.

Finally, networks of random quadrilaterals have been considered. These fractures are generated by randomly injecting four points inside a disk (see Fig. 1 and the Supplemental Material [7]). The resulting fractures have strongly polydisperse sizes and shapes that range from roughly square or roughly triangular to very elongated. This makes this model reminiscent of the variability that can exist in a real fracture network.

Note that the ellipses, lozenges, the most elongated rectangles, the random quadrilaterals, and most of the shape mixtures were not investigated in our earlier contributions. The bidisperse size distributions are also a new addition and the ranges of the ratio $\tilde{R}$ and exponent $a$ for the power law distributions have been widely extended.

\section{B. Dimensionless parameters and percolation threshold}

Some concepts and dimensionless parameters that are used in the data analysis and rationalization are introduced in this section.

Percolation of network on lattices has been extensively studied (see, e.g., [14-16]). These lattices percolate when the probability $p$ of occupation of sites or bonds is larger than a critical value $p_{c}$, called percolation threshold, which depends on the precise lattice structure. When transposed to fracture networks, which pertain to the domain of continuum percolation [17], it is clear that percolation occurs if some threshold density is reached. A quantity equivalent to the probability $p$ in discrete lattices should be found. The excluded volume, first applied in [18] to fracture networks, can be used for this purpose.

The excluded volume $V_{\mathrm{ex}}$ of an object was defined as the volume surrounding it, in which the center of another object must be in order for them to intersect. For randomly oriented and located three-dimensional convex objects $A$ and $B$, the excluded volume is [19]

$$
V_{\mathrm{ex}, A B}=V_{A}+V_{B}+\left(A_{A} R_{B}+A_{B} R_{A}\right),
$$


where $V_{A}$ and $V_{B}$ are the object volumes, $A_{A}$ and $A_{B}$ their surface areas, and $R_{A}$ and $R_{B}$ their mean radii of curvature. For plane convex objects with perimeters $P_{A}$ and $P_{B}, V_{\mathrm{ex}, A B}$ becomes [2]

$$
V_{\mathrm{ex}, A B}=\frac{1}{4}\left(A_{A} P_{B}+A_{B} P_{A}\right) .
$$

When applied to a network where all the fractures are identical, (4) reduces to

$$
V_{\mathrm{ex}}=\frac{1}{2} A P .
$$

Conversely, in a polydisperse network where the fracture characteristics (size and/or shape) are described by a probability distribution $\varphi(F)$, the mean excluded volume obtained by averaging (4) reads

$$
\begin{aligned}
\left\langle V_{\mathrm{ex}}\right\rangle & =\frac{1}{4} \iint \varphi\left(F_{1}\right) \varphi\left(F_{2}\right)\left(A_{1} P_{2}+A_{2} P_{1}\right) d F_{1} d F_{2} \\
& =\frac{1}{2}\langle A\rangle\langle P\rangle,
\end{aligned}
$$

where $\langle\cdot\rangle$ is the statistical average.

The excluded volume provides a natural reference to introduce a dimensionless density $\rho^{\prime}$, defined as the number of objects per volume $V_{\mathrm{ex}}$,

$$
\rho^{\prime}=\rho V_{\mathrm{ex}} .
$$

On the other hand, the definition of $V_{\mathrm{ex}}$ implies that $\rho^{\prime}$ is also the average number of intersections per object, if they are randomly located according to a Poisson process. Therefore, $\rho^{\prime}$ is a direct measure of the network connectivity. For networks containing fractures that do not strongly differ in size or shape, it proved very successful in unifying the values of the percolation threshold $\rho_{c}^{\prime}$ [4]. It is also a powerful tool for the rationalization of many other geometrical or topological properties such as the matrix partition into blocks, and of the network permeability $[12,13,20-22]$.

However, it was observed that whereas $\rho^{\prime}$ is still exactly the mean number of intersection per fracture, it does not govern the global connectivity and the percolation of networks of strongly polydisperse fractures [5]. Intuitively, the reason is that far-reaching connections between large objects are more effective to build percolating clusters than connections between smaller ones. A measure of the network density that accounts for this remark and appropriately weights the different kinds of connections was found in the form of an alternative definition of the dimensionless density. It results from a modification of (6) where $\langle A\rangle\langle P\rangle$ is replaced by $\langle A P\rangle$, i.e.,

$$
\rho_{3}^{\prime}=\rho \frac{1}{2}\langle A P\rangle .
$$

This definition can be applied to mixtures of fractures with any sizes and shapes, and reduces of course to $\rho^{\prime}$ if all the fractures in the network are identical. The subscript " 3 " is a reminder that $\langle A P\rangle$ in (8) scales as the third moment of the fracture sizes, while $\langle A\rangle\langle P\rangle$ in the definition of $\rho^{\prime}$ scales as the product of their first and second moments. This dimensionless density was found very successful to describe the percolation and flow properties of networks of fractures with identical shapes and polydisperse sizes with a power law distribution [23]. The reduced variance $\Sigma_{A P}^{2}$ of the product $A P$ is used in the following to quantify the degree of polydispersity of a network. It is defined by

$$
\Sigma_{A P}^{2}=\frac{\left\langle(A P)^{2}\right\rangle-\langle A P\rangle^{2}}{\langle A P\rangle^{2}} .
$$

Finally, let us introduce for later use the three following shape factors that measure in different ways the departure of a flat, convex object shape from circularity:

$$
\begin{aligned}
& \tilde{\eta}_{P}=\frac{4 R / P-2 / \pi}{1-2 / \pi}, \quad \tilde{\eta}=1-4 \pi \frac{A}{P^{2}}, \\
& \tilde{\eta}_{g}=1-\sqrt{\frac{A}{2 \pi R_{g}^{2}}} \quad \text { with } R_{g}^{2}=\frac{1}{A} \int_{A} r^{2} d s,
\end{aligned}
$$

where $A, P$, and $R$ are the object surface area, perimeter, and bounding radius, i.e., the radius of its minimum bounding circle. The gyration radius $R_{g}$ is the quadratic average of the distance $r$ of a point in the object from its barycenter. These quantities are given in Table I for all the fracture shapes considered in this work. The three shape factors are normalized so that they all range from 0 for a disk to 1 for very elongated shapes with vanishing area. The first one $\tilde{\eta}_{P}$ was already used in $[5,12]$ to account for the effect of the fracture shape elongation on the network percolation properties. The isoperimetric deficit $\tilde{\eta}$ is a classical indicator in applied morphological analysis, which proved to be a relevant parameter for other topological properties of the network [22]. We are not aware of any prior use of $\tilde{\eta}_{g}$ in the literature. For polydisperse networks, a mean value of $\tilde{\eta}_{g}$ will be used in the following, which results from a rms average weighted by the

TABLE I. Geometrical characteristics of various shapes with unit bounding radius. $K$ and $E$ are the complete elliptic integrals of the first and second kinds. The aspect ratio is the ratio of the major and minor semiaxes $(f \geqslant 1)$.

\begin{tabular}{lcccc}
\hline \hline Object shape & $A$ & $P$ & $R_{g}^{2}$ & $R_{h}^{2} / R_{g}^{2}$ \\
\hline$n$-sided regular polygons & $n \sin \frac{\pi}{n} \cos \frac{\pi}{n}$ & $2 n \sin \frac{\pi}{n}$ & $\frac{1}{6}\left(2+\cos \frac{2 \pi}{n}\right)$ & 2 \\
Ellipses with aspect ratio $f$ & $\frac{\pi}{f}$ & $4 E\left(1-\frac{1}{f^{2}}\right)$ & $\frac{1}{4}\left(1+\frac{1}{f^{2}}\right)$ & $\frac{4}{3}\left[1+\frac{1}{1+f^{2}} \frac{K\left(1-\frac{1}{f^{2}}\right)}{E\left(1-\frac{1}{f^{2}}\right)}\right]$ \\
Rectangles with aspect ratio $f$ & $\frac{4 f}{1+f^{2}}$ & $\frac{4(1+f)}{\sqrt{1+f^{2}}}$ & $1 / 3$ & $\frac{(1+f)^{2}}{1+f^{2}}$ \\
Lozenges with aspect ratio $f$ & $\frac{2}{f}$ & $4 \sqrt{1+\frac{1}{f^{2}}}$ & $\frac{1}{4}\left(1+\frac{1}{f^{2}}\right)$ & 2 \\
\hline
\end{tabular}


product $A P$ of the fractures, i.e.,

$$
\left\langle\tilde{\eta}_{g}\right\rangle=\left\{\frac{\left\langle A P\left(1-\sqrt{A / 2 \pi R_{g}^{2}}\right)^{2}\right\rangle}{\langle A P\rangle}\right\}^{1 / 2} .
$$

\section{Determination of the percolation threshold}

The percolation threshold of fracture networks is determined in a similar way as in earlier works [4,5]. The main steps of the procedure are recalled briefly, and only some minor differences are discussed in more detail.

Recall that the generated networks are spatially periodic, with a unit cell of size $L^{3}$. It can easily be determined whether any two fractures intersect, and a graph, denoted by $\Gamma_{1}$, is built from this knowledge. $\Gamma_{1}$ summarizes all the connections in the network. Its vertices and edges correspond to the fractures and to the intersection between fractures, respectively. Due to the periodicity, two fractures in neighboring cells can be connected across the cell boundaries.

The sets of connected fractures, or connected clusters, can be determined from $\Gamma_{1}$. As the density $\rho$ of fractures increases, their typical size $\xi$ increases and at some point, an infinite cluster develops that spans the whole system. This corresponds to the onset of percolation. In a periodic network, it occurs as soon as a cluster contains two replicas of the same fracture in different cells.

The transition to percolation in an infinite medium is a critical process. It occurs with probability 1 at a critical density $\rho_{c}$ which depends on the system contents (fracture shapes and sizes). If the medium is statistically isotropic, it also occurs simultaneously along all the directions. But in finite systems or in periodic media with a finite cell size $L$, the transition is smooth. The percolation probability $\Pi_{L}(\rho)$ for a random realization of a system of size $L$ increases with the density $\rho$, with a transition from 0 to 1 over a range of the order of $\Delta_{L}$.

This increase is generally described by an error function of the form

$$
\Pi_{L}(\rho)=\frac{1}{\sqrt{2 \pi} \Delta_{L}} \int_{-\infty}^{\rho} \exp \left\{-\frac{\left(\xi-\rho_{L c}\right)^{2}}{2\left(\Delta_{L}\right)^{2}}\right\} d \xi,
$$

where $\rho_{L c}$ is the density for which $\Pi_{L}(\rho)=1 / 2$. As $L$ increases, the transition becomes sharper, $\Delta_{L}$ decreases and $\rho_{L c}$ converges toward the limit $\rho_{c}$ for infinite systems. These size effects are described by the classical scaling laws [15]

$$
\rho_{L c}-\rho_{c} \propto L^{-1 / v}, \quad \Delta_{L} \propto L^{-1 / v} .
$$

In addition, percolation in finite systems generally occurs first along some direction, and only later along the other ones. Therefore, the probability $\Pi_{L}$ can be defined according to various criteria: $\Pi_{L}^{(1)}$ for percolation along at least one direction, $\Pi_{L}^{(x)}$ for percolation along a prescribed direction (say $x$ ), and $\Pi_{L}^{(3)}$ for percolation along the three directions $x$, $y$, and $z$. Each of them can be described by (12), with different scaling laws (13) although they all converge to the same limit $\rho_{c}$. Illustrations of these features are provided in Fig. 2.

In view of this, the following procedure is implemented for the determination of the percolation threshold $\rho_{c}$ for networks made of fractures with any prescribed characteristics (shapes, sizes). Note that it can be implemented indifferently in terms of the densities $\rho$ or $\rho_{3}^{\prime}$, which are related by a constant factor [see Eq. (8)].

(a) For a given cell size $L$, choose a set of $\mathcal{N}_{\rho}$ values $\rho$ evenly distributed in the expected range of transition of $\Pi_{L}$ from nearly 0 to nearly 1 .

(b) For each value of $\rho$, generate a set of $\mathcal{N}_{r}$ random realizations, check their percolation status, and get an estimate of the probability $\Pi_{L}(\rho)$.

(c) Determine $\rho_{L c}$ and $\Delta_{L}$ by a least square fit of $\Pi_{L}(\rho)$ by the error function (12).

(d) Use the scaling laws (13) to estimate $\rho_{c}$ from the linear extrapolation of $\rho_{L c}$ as a function of $\Delta_{L}$, when $\Delta_{L} \rightarrow 0$ (a

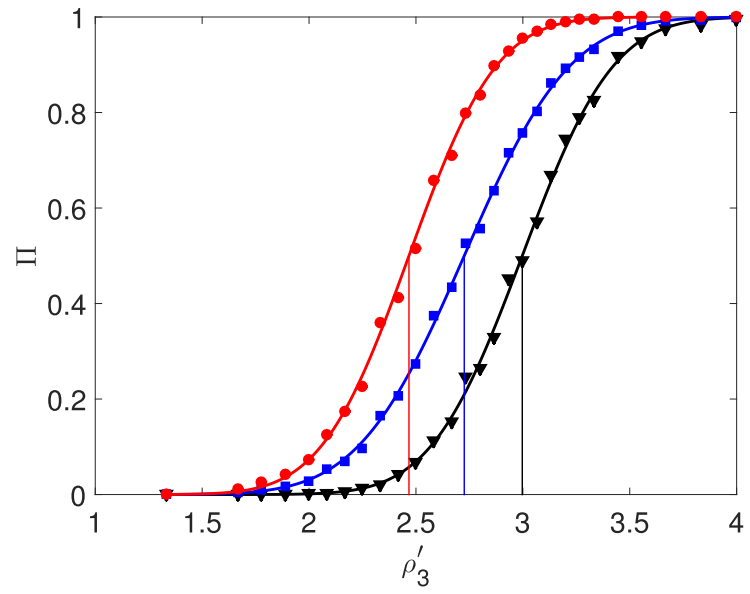

(a)

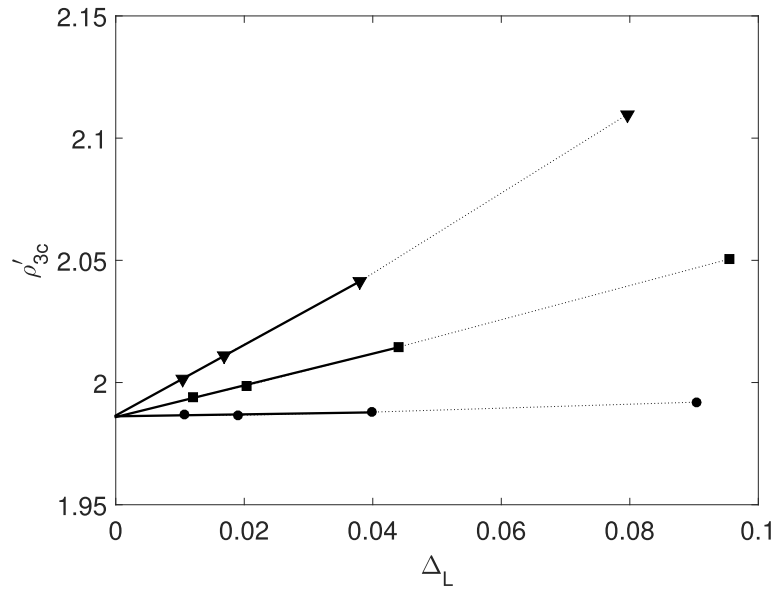

(b)

FIG. 2. (a) The percolation probabilities $\Pi_{L}^{(1)}\left(\right.$ red, $\bullet$ ), $\Pi_{L}^{(x)}$ (blue, $\left.\mathbf{\square}\right)$, and $\Pi_{L}^{(3)}$ (black, $\mathbf{\nabla}$ ) as functions of $\rho_{3}^{\prime}$ for networks of bidisperse disks with $\tilde{R}=6, \varphi_{M}=0.04$, and $L / R_{M}=8$. The curves correspond to the fit (12) and the vertical lines indicate the positions of $\rho_{L c}^{(1)}, \rho_{L c}^{(x)}$, and $\rho_{L c}^{(3)}$. (b) The densities $\rho_{L 3 c}^{\prime(1)}(\bullet), \rho_{L 3 c}^{\prime(x)}(\mathbf{\square})$, and $\rho_{L 3 c}^{\prime(3)}(\boldsymbol{\nabla})$ as functions of the corresponding $\Delta_{L}$ for networks containing $75 \%$ of disks and $25 \%$ of eight-ellipses with the same area. The cell size $L$ ranges from 10.3 to 61.8 times the semimajor axis of the ellipses (right to left). The heavy lines are linear fits which yield the extrapolated values $1.9862,1.9859$, and 1.9865 for $\Delta_{L}=0(L \rightarrow \infty)$. 
two-step fit where $v$ is determined from the scaling of $\Delta_{L}$ with $L$ and then $\rho_{c}$ from the scaling of $\rho_{L c}$ with $L^{-1 / v}$ yields equivalent results).

The three percolation criteria are applied in step (b) to determine $\Pi_{L}^{(1)}, \Pi_{L}^{(x)}$, and $\Pi_{L}^{(3)}$ and the following steps (c) and (d) are performed based on each of these data, which provides three estimates $\rho_{c}^{(1)}, \rho_{c}^{(x)}$, and $\rho_{c}^{(3)}$ of $\rho_{c}$. They should be identical and their scatter is an indication of the prediction uncertainty.

The extensive calculations involved in this procedure are costly, and careful choices of the range of cell size $L$, the range and number $\mathcal{N}_{\rho}$ of investigated densities, and the number $\mathcal{N}_{r}$ of realizations have to be made in order to achieve a good accuracy without wasting resources. Accumulated experience resulted in the following choices: $\mathcal{N}_{\rho} \sim 20$ values of $\rho$ are considered in a range that is suggested by the results of earlier calculations for a smaller value of $L$, if available, and by the predictive models presented in the following if a yet unexplored kind of network is addressed. The number or realizations $\mathcal{N}_{r}$ is generally 500 for the polydisperse network, $100-500$ for the mixtures of shapes with similar sizes, and it can be reduced to 50 when the variability is small because the fractures in the networks are all identical, with very large cell sizes.

Finally, increasing cell sizes $L$ up to $L_{\max }$ have been investigated. The values of $L_{\max } / R_{\max }$, where $R_{\max }$ is the largest bounding radius of the fractures in the network, are reported in the detailed tables of the Supplemental Material [7]. Large values of the order of 100 have been used for the monodisperse networks since the computations are relatively fast. Smaller values are sufficient in polydisperse networks. $L_{\max } / R_{\max }$ was kept larger than 20 , or exceptionally 10 in a few cases of power law size distributions with large exponents since these networks actually contain very few fractures of size $R_{\max }$.

Note that the pattern observed in the example of Fig. 2(b) with a very weak dependence of $\rho_{L c}^{(1)}$ on $L$ is a general feature. Therefore, $\rho_{L c}^{(1)}$ provides a fairly accurate estimation of $\rho_{c}$ even without extrapolation. Advantage of this was taken in some cases. The extrapolation step (d) was skipped, when it was known from comparable situations that $\rho_{L c}^{(1)}$ for some value of $L$ provides an adequate approximation of $\rho_{c}$. Thus, the data used in the discussion and given in the fully detailed set of results provided in the Supplemental Material [7] are generally the average of the three extrapolated values $\rho_{c}^{(1)}$, $\rho_{c}^{(x)}$, and $\rho_{c}^{(3)}$, or in some cases indicated in Tables VII and VIII in the Supplemental Material [7], the value $\rho_{L c}^{(1)}$ obtained with the specified size $L$ without extrapolation.

Note finally that in view of (8), the number $\mathcal{N}_{\text {fr }}$ of fractures in each realization of network for $\rho \sim \rho_{3 c}$ is about $2 \rho_{3}^{\prime} L^{3} /\langle A P\rangle$. With the largest investigated cell sizes, typical values of $\mathcal{N}_{\text {fr }}$ are in the ranges $10^{5}-10^{6}$ for most monodisperse cases and shape mixtures (Tables I and II in the Supplemental Material [7]), $\sim 10^{4}\left(\right.$ when $\left.\varphi_{M} \sim 1\right)$ to $\sim 10^{6}\left(\right.$ when $\left.\varphi_{M} \ll 1\right)$ for bidisperse networks (Table IV in the Supplemental Material [7]), and $\sim 10^{4}$ (for small $a$ ) to $\sim 10^{5}$ (for large $a$ ) for power law size distributions (Table VII in the Supplemental Material [7]). $\mathcal{N}_{\text {fr }}$ can exceed $3 \times 10^{6}$ in some cases with elongated ellipses or when exceptionally large cells are used to thoroughly check the size scaling effects.
The results can be subject to two kinds of uncertainties, resulting from statistical noise and from an inadequate correction for the finite size effects. The former contribution is difficult to assess a priori, in view of the many successive operations involved in steps (a)-(c), but it can be estimated by comparing the results of several predictions from separate data sets, for instance by splitting the $\mathcal{N}_{r}$ realizations into four subsets and processing them separately. A more robust estimator is obtained by considering 100 subsets of $\mathcal{N}_{r} / 4$ randomly picked realizations and measuring the standard deviation $\sigma_{\rho_{L c}}$ of the resulting threshold values $\rho_{L c}$. In terms of $\rho_{3 c}^{\prime}$ and with the largest domain size $L_{\max }, \sigma_{\rho_{L c}}<0.005$ in nearly all cases and never exceeds 0.007 .

As already noted, defects in the correction for finite size effects can be detected and quantified by the scatter of $\rho_{c}^{(1)}, \rho_{c}^{(x)}$, and $\rho_{c}^{(3)}$. As a rule, each of these values does not differ from their average $\rho_{c}$ by more than \pm 0.002 for monodisperse networks, \pm 0.003 for mixtures of fractures with different shapes, and \pm 0.010 for networks of fractures with polydisperse sizes, in terms of $\rho_{3 c}^{\prime}$, but larger deviations occur occasionally. They probably result in part from statistical fluctuations in addition to residual size effects, but in order to stay on the safe side, we regard them as independent sources of errors and define the confidence interval of the data by summing the two contributions,

$$
\max _{i=1, x, 3}\left|\rho_{c}^{(i)}-\rho_{c}\right|+2 \sigma_{\rho_{\max }} .
$$

This quantity is systematically reported in the detailed tables of the Supplemental Material [7]. It is always smaller than 0.01 for monodisperse and weakly polydisperse networks, but often ranges between 0.01 and 0.02 for strongly polydisperse networks, and exceptionally up to 0.03 . When the extrapolation step was skipped, the first contribution is not available and (14) is replaced by a conservative value of 0.02 , based on similar or more demanding cases (see Tables VI-VIII in the Supplemental Material [7]).

\section{NETWORKS OF IDENTICAL FRACTURES}

We consider here networks of identical fractures, i.e., the networks denoted $\mathrm{I}^{2}$ OUD in [12], with a variety of shapes (results in Table I in the Supplemental Material [7]). We first addressed this case in [4] and revisited it in later works [5,12] where a greater variety of situations was explored. A still wider range of fracture shapes is considered here, with the addition of ellipses and lozenges. The precision of the data was also improved, but the values reported here and displayed in Fig. 3 differ only marginally from the earlier ones, when available.

When these results are analyzed in terms of the average number of intersections per fracture $\rho^{\prime}$, with a different excluded volume for each shape, a remarkable property stands out. As already noted in [4], the dimensionless percolation threshold appears to be independent of the fracture shape, with

$$
\rho_{c}^{\prime} \approx 2.29 \pm 0.05
$$

for all regular polygons, disks, and two-rectangles. It should be emphasized that other measures yield much more scattered values. For instance, if the volume $R^{3}$ based 


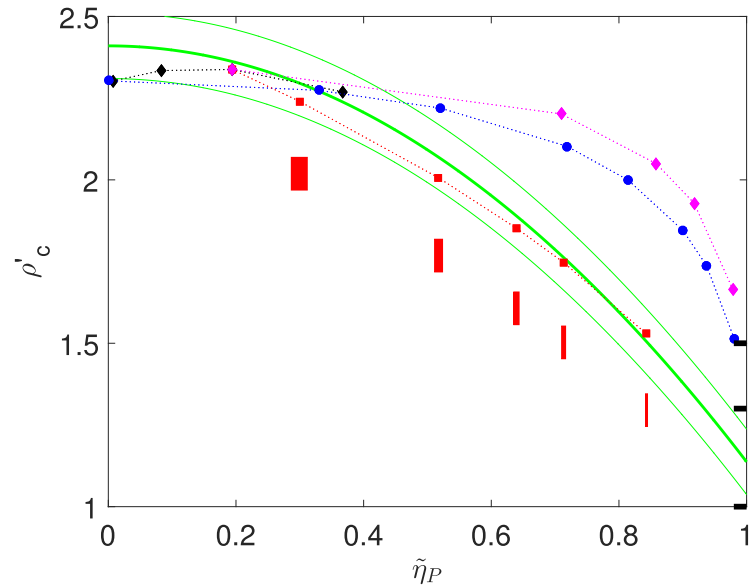

(a)

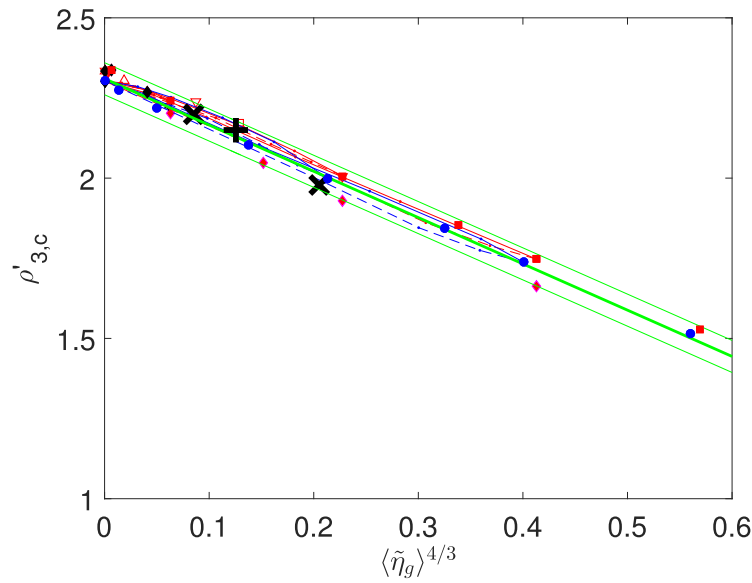

(c)

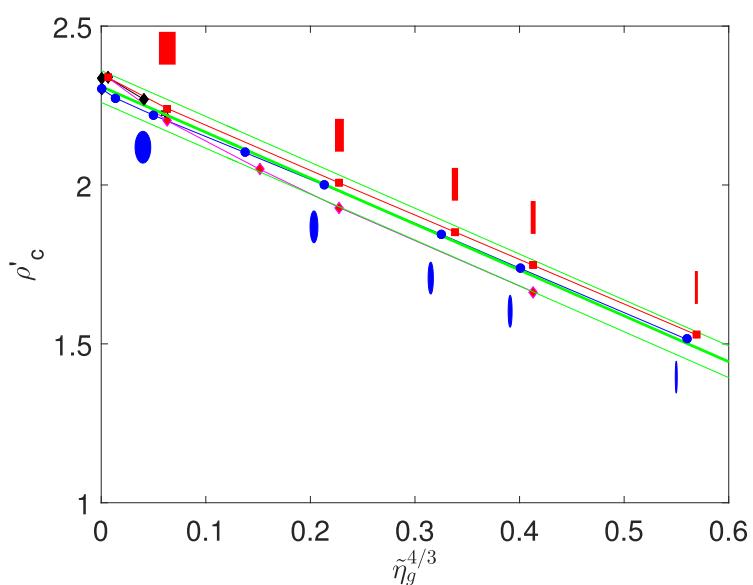

(b)

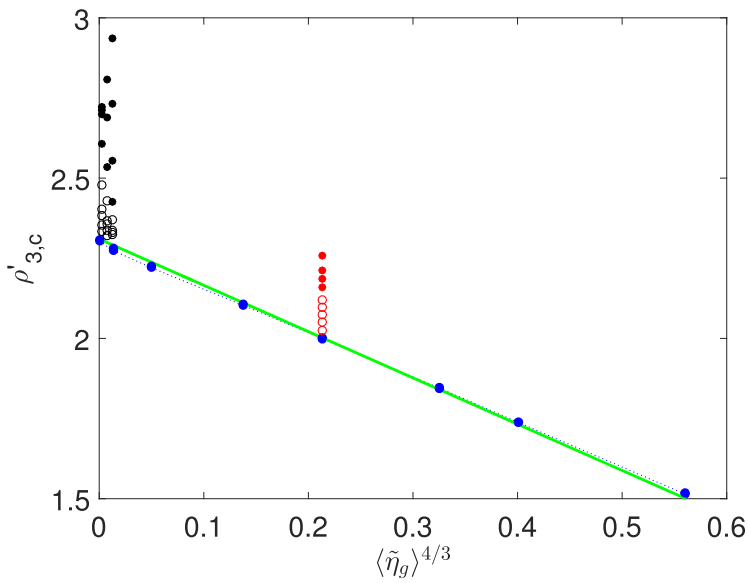

(d)

FIG. 3. Percolation thresholds $\rho_{c}^{\prime}$ as functions of $\tilde{\eta}_{P}$ (a) or $\tilde{\eta}_{g}$ (b) and $\rho_{3 c}^{\prime}$ as a function of $\left\langle\tilde{\eta}_{g}\right\rangle$ (c). Data in (a),(b) are for networks of identical fractures with various shapes: disks and regular polygons (black $\downarrow$ ), rectangles (red, $\mathbf{\square})$, lozenges (magenta, $\bullet$ ), and ellipses (blue, $\bullet$ ). The icons depict the shapes of the rectangles in (a) and (b) and ellipses in (b). In addition, the data in (c) include binary mixtures in various proportions of 20-gons + rectangles (red, — - — for equal bounding radii and - - . - - for equal area) and disks + ellipses (blue, - . - for equal bounding radii and - - - - for equal area), mixtures in equal proportions of hexagons + triangles (red, $\boldsymbol{\Lambda}$ ), hexagons + four-rectangles (red, -) and squares + eight-rectangles (red, $\mathbf{\square})$, ternary mixtures of disks + three-ellipses + nine-ellipses $(x)$ and networks of random quadrilaterals (+). Data in (d) are for monodisperse disks and ellipses (blue), four-ellipses with $a=4$ (red, solid symbols if $\tilde{R} \geqslant 8$ ) and bidisperse disks with $\tilde{R} \geqslant 5$ (black, solid symbols if $\varphi_{M} \leqslant 0.02$, slightly shifted horizontally for readability). The marks on the right in (a) are the predictions of [24-26] for infinitely elongated objects. The solid lines correspond to the models and error bars (16) in (a), (17) in (b), and (18) in (c) and (d).

on the bounding radius $R$ of the fractures is used as a reference, $\rho_{c} R^{3}$ ranges from 0.234 (disks) to 0.672 (triangles). Thus, (15) demonstrates the success of the excluded volume in accounting for the fracture shape effects.

Calculations including $f$-rectangles with $f$ up to 16 showed that a correction to (15) is required for very slender fracture shapes. All the data in $[5,12]$ could be summarized by a formula which can be expressed in terms of the shape factor $\tilde{\eta}_{P}$

$$
\rho_{c}^{\prime}=2.41\left[1-0.53 \tilde{\eta}_{P}^{2}\right] \pm 0.10 .
$$

This relation is illustrated in Fig. 3(a). While it successfully describes the data of [5], it appears that it does not accommodate slender ellipses and lozenges, which were investigated only later. Therefore, a more general and robust model had to be formulated. The shape factor $\tilde{\eta}$ based on the ratio $A / P^{2}$ was considered, but did not yield a satisfactory fit, although it has proved to be a relevant parameter for other topological properties of the network [22]. A much better description is obtained in terms of the last shape factor $\tilde{\eta}_{g}$ in (10).

This shape factor is based on the gyration radius $R_{g}$ of the fracture shape. $R_{g}$ was used by Alon et al. [6] in their argument for the prediction of the threshold in continuous percolation, as an estimator for the typical "bonding distance." Intuitively, far-reaching intersecting objects with large $R_{g}$ are more efficient to increase a connected cluster size than say, disks with the same area. Hence, the ratio of the fracture area to $R_{g}^{2}$ is a good candidate. An extremely good fit of all the data for $\mathrm{I}^{2}$ OUD networks is provided by the model illustrated in 


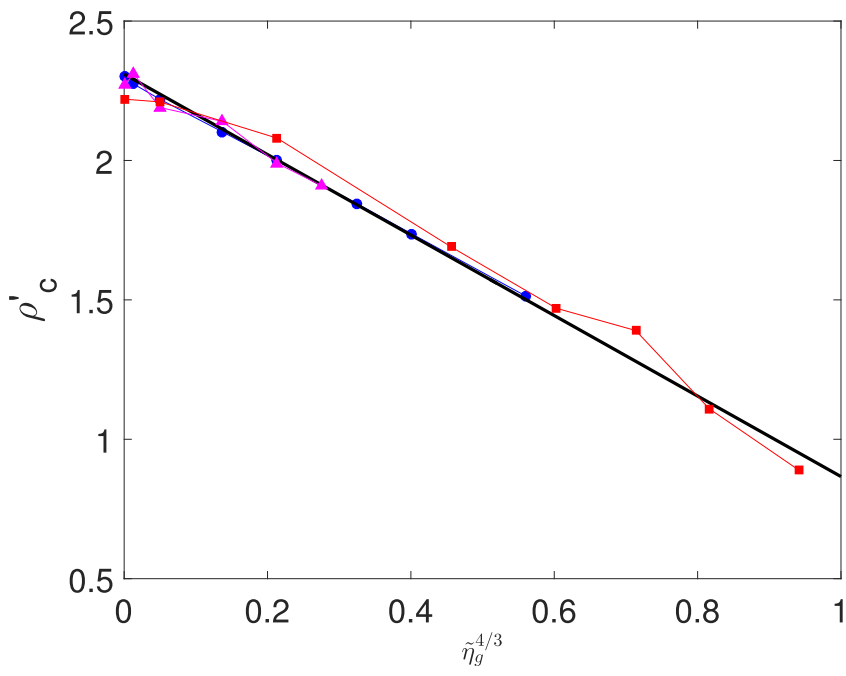

FIG. 4. The percolation threshold $\rho_{c}^{\prime}$ as a function of $\tilde{\eta}_{g}$ for networks of identical ellipses. Symbols correspond to the data of [27] $(\boldsymbol{\square})$, of [28] ( $\mathbf{\Delta})$, and to the present calculations $(\bullet)$. The thick straight line corresponds to the model (17).

Fig. 3(b),

$$
\rho_{c, r}^{\prime}=2.31\left[1-\frac{5}{8} \tilde{\eta}_{g}^{4 / 3}\right] \pm 0.05 .
$$

Data for networks of ellipses from the literature are compared to ours in Fig. 4. Networks of ellipses with very large aspect ratios up to $f=1000$ have been investigated in [27], with monodisperse or power law size distributions. Unfortunately, the discussion of the results was smeared by a mistake in the evaluation of the excluded volume, but a correction can be made in the monodisperse cases. The corrected data are in good agreement with the present results. The deviations do not exceed \pm 0.1 from our numerical results when available, and from (17) for values of $f$ larger than investigated here. The data of [28] for $1 \leqslant f \leqslant 5$ are also in excellent agreement with our data.

\section{MIXTURES OF FRACTURES WITH DIFFERENT SHAPES AND SIMILAR SIZES}

We consider here networks of fractures with different shapes. Only a few such cases had been addressed in earlier works. Mixtures in various proportions of 20 -gons with $f$ rectangles $(f=1-8)$ and of disks with four- and eight-ellipses are systematically examined here (Table II in the Supplemental Material [7]). The two kinds of fractures are always set with identical bounding radii, or with identical areas. For instance in the latter case, the diameter of the eight-ellipses is larger than the disk diameter by a factor of $2 \sqrt{2}$. A few additional mixtures include hexagons and triangles, hexagons and four-rectangles, and squares and eight-rectangles, in equal proportions and with equal bounding radii. Ternary mixtures of disks, three-ellipses, and nine-ellipses with identical bounding radii have also been considered (Table III in the Supplemental Material [7]), in equal proportions $(1 / 3,1 / 3,1 / 3)$, and in proportions $(1 / 13$, $3 / 13,9 / 13$ ) so that the volumetric areas of each kind of fracture are identical. Finally, the networks of random quadrilaterals (Table III in the Supplemental Material [7]) contain fractures with a great variety of shapes and a wider range of sizes than the other mixtures.

The discussion is very simple, as shown by Fig. 3(c) where all these data are displayed. Since the networks possibly contain very different kinds of fractures, the percolation threshold is expressed in terms of $\rho_{3 c}^{\prime}$ and the mean shape factor $\left\langle\tilde{\eta}_{g}\right\rangle$ is used as defined in (11). In these terms, the data for all the mixtures comply with the same model as for networks of identical fractures, i.e.,

$$
\rho_{3 c}^{\prime}=2.31\left[1-\frac{5}{8}\left\langle\tilde{\eta}_{g}\right\rangle^{4 / 3}\right] \pm 0.05 .
$$

Note for future reference that for bidisperse mixtures, the reduced variance $\Sigma_{A P}^{2}(9)$ reads

$$
\Sigma_{A P}^{2}=\varphi_{M} \varphi_{m}\left[\frac{(A P)_{M}-(A P)_{m}}{\varphi_{M}(A P)_{M}+\varphi_{m}(A P)_{m}}\right]^{2},
$$

where $(A P)_{M}$ and $(A P)_{m}$ are the largest and smallest values of $A P$, with associated fractions $\varphi_{M}$ and $\varphi_{m}=1-\varphi_{M}$. The values of $\Sigma_{A P}$ for all the mixtures considered in this section are given in Tables II and III in the Supplemental Material [7]. Its maximum $\Sigma_{A P_{\max }}$ is reached when $\varphi_{M}(A P)_{M}=\varphi_{m}(A P)_{m}$, i.e.,

$$
\begin{aligned}
\varphi_{M_{\max }} & =\frac{1}{1+(A P)_{M} /(A P)_{m}}, \\
\Sigma_{A P_{\max }}^{2} & =\frac{\left[(A P)_{M}-(A P)_{m}\right]^{2}}{4(A P)_{M}(A P)_{m}} .
\end{aligned}
$$

\section{NETWORKS OF FRACTURES WITH POLYDISPERSE SIZES}

\section{A. Preliminary remarks}

A few illustrative examples of results for polydisperse networks are displayed in Fig. 3(d), including bidisperse disks with $\tilde{R} \geqslant 5$ and four-ellipses with a power law distribution of sizes with exponent $a=4$. It appears that the combination of a large size contrast ratio $\tilde{R}$ with some mixture compositions (large exponent $a$ or $\varphi_{M}$ in a narrow unfavorable range) can lead to significant deviations from the model (18). These situations were not explored in [5] and therefore, it was concluded that the modified dimensionless density $\rho_{3}^{\prime}$ incorporates all the influence of the size polydispersivity.

This is true in a large respect. In the worst cases of Fig. 3(d), $\rho_{3 c}^{\prime}$ in the polydisperse and corresponding monodisperse networks differ by about $12 \%$ (four-ellipses, power law) or $22 \%$ (bidisperse disks), whereas in terms of $\rho_{c}^{\prime}$ these thresholds differ by a ratio of at least 2 (and by a ratio of several hundreds if the comparison is made in terms of $\rho_{c} R_{M}^{3}$ ). Thus, (18) at least provides a reasonable approximation of the percolation threshold in polydisperse networks, with a fairly good accuracy for mild polydispersity. The purpose of this section is to identify the circumstances that cause a significant deviation, and to evaluate and possibly model the required correction to (18).

For this purpose, two size distributions have been investigated, namely the bidisperse and power law distributions introduced in Sec. A. Bidisperse networks of disks have been systematically studied by varying the size ratio $\tilde{R}$ and the fraction $\varphi_{M}$ of larger disks. Other shapes such as four-ellipses, 


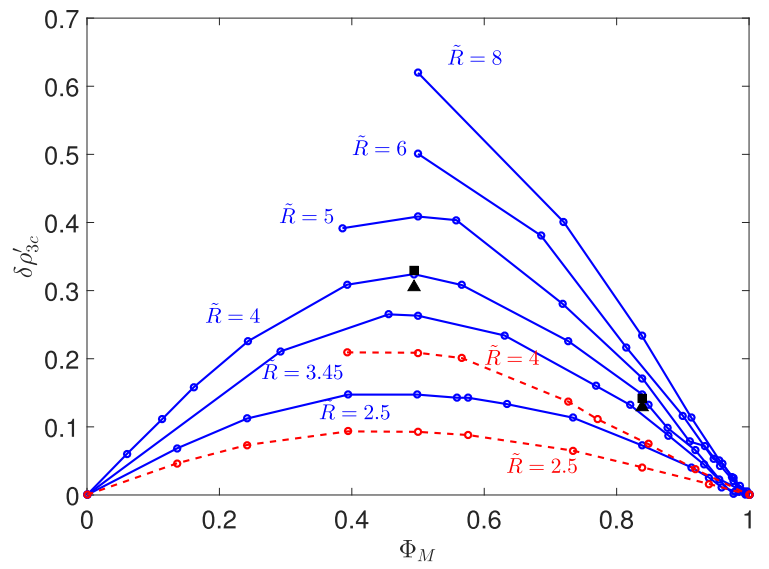

(a)

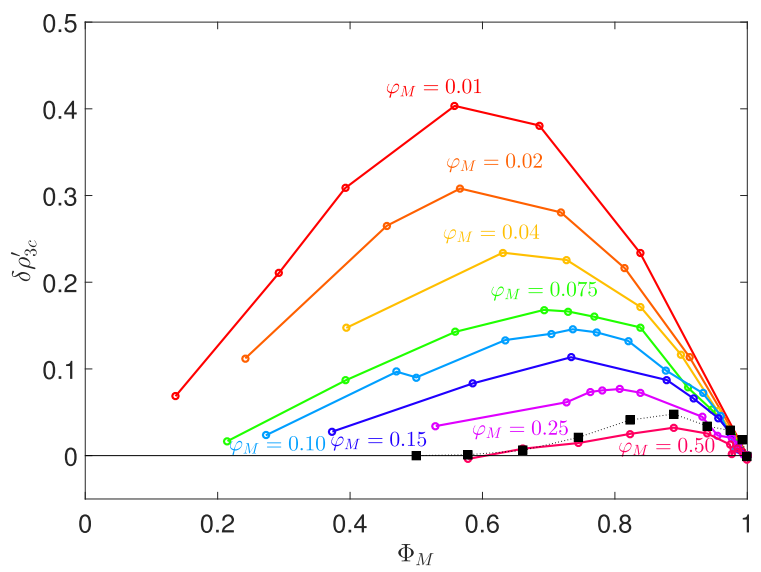

(b)

FIG. 5. The increment $\delta \rho_{3 c}^{\prime}$ in bidisperse networks as a function of the composition parameter $\Phi_{M}$. The curves in (a) are for constant ratios $\tilde{R}$ and varying $\varphi_{M}$. The curves in (b) are for constant $\varphi_{M}$ and varying ratios $\tilde{R}$. Solid lines correspond to disks and broken lines to four-ellipses. Black symbols in (a) correspond to squares ( $\boldsymbol{\square})$ and triangles $(\boldsymbol{\Lambda})$ with $\tilde{R}=4$. Black symbols in (b) (ם) correspond to the data of [28] for disks with $\varphi_{M}=0.50$.

and regular polygons have been examined in a less extensive range (Tables IV-VI in the Supplemental Material [7]). The same shapes have been considered for power law distributions, in a wide range of size ratio $\tilde{R}$ and exponent $a$ (Tables VII and VIII in the Supplemental Material [7]).

The first general observation is that polydispersity always increases the value of $\rho_{3 c}^{\prime}$ with respect to that $\rho_{3 c}^{\prime(m)}$ for the monodisperse network of fractures with the same shape. For this reason, the data are analyzed in terms of the increment $\delta \rho_{3 c}^{\prime}$,

$$
\delta \rho_{3 c}^{\prime}=\rho_{3 c}^{\prime}-\rho_{3 c}^{\prime(m)},
$$

which is also the corrective term to be applied to (18) for polydisperse networks.

\section{B. Bidisperse networks}

The results for bidisperse networks are presented in Fig. 5 as functions of the composition, quantified by the fraction $\Phi_{M}$ of larger objects weighted by their cubed size, as suggested by (8),

$$
\Phi_{M}=\frac{\varphi_{M} R_{M}^{3}}{\varphi_{M} R_{M}^{3}+\left(1-\varphi_{M}\right) R_{m}^{3}}, \quad \Phi_{m}=1-\Phi_{M} .
$$

Note first that the increment $\delta \rho_{3 c}^{\prime}$ for networks of regular polygons (squares and triangles) is nearly identical to that for networks of disks with the same size distribution. However, the effect of size polydispersity is weaker for the elongated four-ellipses than for disks [Fig. 5(a)].

When the size ratio $\tilde{R}$ is kept constant, $\delta \rho_{3 c}^{\prime}$ is maximum when $\Phi_{M}=\Phi_{m}=1 / 2$. This corresponds to a proportion $\varphi_{M_{\max }}$ of larger objects equal to

$$
\varphi_{M_{\max }}=\frac{1}{1+\tilde{R}^{3}} .
$$

This fraction becomes very small when the size contrast is large. When $\varphi_{M} \gg \varphi_{M_{\max }}$, the connectivity and percolation of the networks is dominated by the large fractures and $\delta \rho_{3 c}^{\prime}$ is very small [Fig. 5(b)]. For instance, $\delta \rho_{3 c}^{\prime}$ never exceeds 0.04 if the small and large fractures are in equal numbers. Our data for this case are found in good agreement with those of [28] for $\varphi_{M}=0.5$ and $\tilde{R}$ up to 10 [Fig. 5(b)]. It should be noted however that $\delta \rho_{3 c}^{\prime}$ was evaluated relative to $\rho_{3 c}^{\prime(1)}$ which is found equal to 2.265 in [28], lower than our result 2.303 by 0.038 .

In bidisperse networks, the fraction $\varphi_{M_{\max }}$ also corresponds to the composition that maximizes the normalized variance $\Sigma_{A P}^{2}(9)$ of the product $A P$,

$$
\Sigma_{A P}^{2}=\frac{\varphi_{M}\left(1-\varphi_{M}\right)\left(\tilde{R}^{3}-1\right)^{2}}{\left[\varphi_{M} \tilde{R}^{3}+\left(1-\varphi_{M}\right)\right]^{2}}
$$

with

$$
\Sigma_{A P_{\max }}^{2}(\tilde{R})=\Sigma_{A P}^{2}\left(\tilde{R}, \varphi_{M_{\max }}\right)=\frac{\left(\tilde{R}^{3}-1\right)^{2}}{4 \tilde{R}} .
$$

The plot of $\delta \rho_{3 c}^{\prime}$ vs $\Sigma_{A P}^{1 / 2}$ in Fig. 6 for networks of bidisperse disks confirms that the increment is indeed maximal when $\Sigma_{A P}$ is maximal, i.e., when $\varphi_{M}$ corresponds to (23), for any size ratio $\tilde{R}$. In addition, the maximal increment can be represented by the linear expression

$$
\begin{aligned}
\delta \rho_{3 c_{\max }}^{\prime}= & \alpha\left[\Sigma_{A P_{\max }}^{1 / 2}-\left(\frac{1}{2}\right)^{1 / 2}\right] \quad \text { with } \alpha=\frac{1}{4} \\
& \text { (bidisperse disks, } \left.\Sigma_{A P_{\max }} \geqslant 1 / 2\right) .
\end{aligned}
$$

Furthermore, $\delta \rho_{3 c}^{\prime}$ for other values of $\varphi_{M}$ is very well represented by the cubic law

$$
\delta \rho_{3 c}^{\prime}=\delta \rho_{3 c_{\max }}^{\prime}(\tilde{R})\left[\frac{\Sigma_{A P}}{\Sigma_{A P_{\max }}(\tilde{R})}\right]^{3} .
$$

Hence, a full predictive model is obtained by combining (25)(27), which approximates all numerical data for bidisperse networks of disks within \pm 0.04 with a rms deviation equal to 0.015 . Since the increment $\delta \rho_{3 c}^{\prime}$ for regular polygons is found very close to that for disks [Fig. 5(a)], this model also applies to networks of fractures with regular polygonal shapes, and probably with any shapes that do not strongly deviate from circularity. 


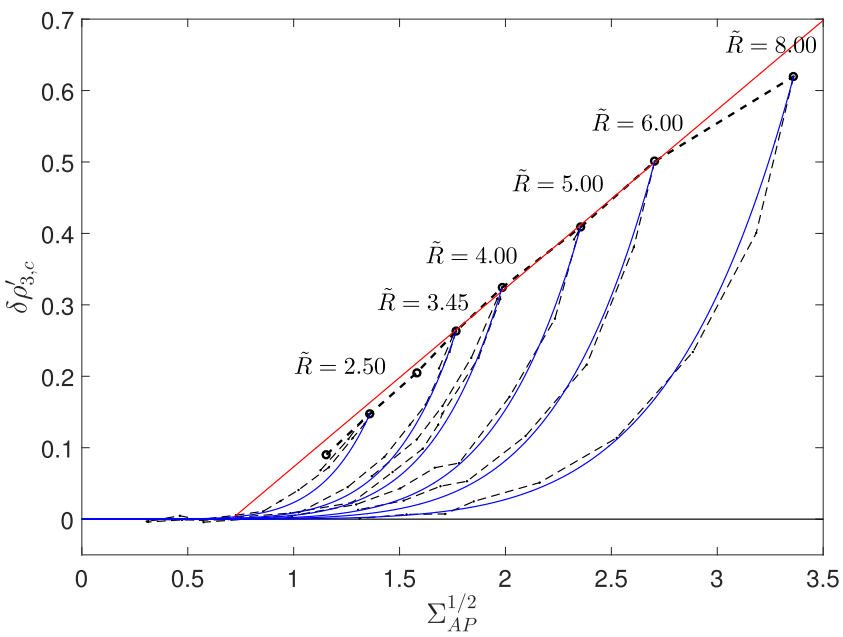

FIG. 6. The increment $\delta \rho_{3 c}^{\prime}$ in bidisperse networks of disks as a function of $\Sigma_{A P}^{1 / 2}$. The data correspond to constant ratios $\tilde{R}$ with varying $\varphi_{M}(--.--)$ and to various $\tilde{R}$ with $\varphi_{M}=\varphi_{M_{\max }}(--\circ--)$. The red straight line and the blue solid curves correspond to (26) and (27), respectively.

However, elongated fractures seem to behave differently, as seen in Fig. 5(a) where four-ellipses yield smaller increments than disks. Nevertheless, the maximum increment is still reached for the composition (23) that maximizes $\Sigma_{A P}$ and the value of $\delta \rho_{3 c_{\max }}^{\prime}$ still varies according to (26), with a modified value of $\alpha=1 / 6$, as illustrated in Fig. 8(a),

$$
\begin{aligned}
\delta \rho_{3 c_{\max }}^{\prime}= & \alpha\left[\Sigma_{A P_{\max }}^{1 / 2}-\left(\frac{1}{2}\right)^{1 / 2}\right] \quad \text { with } \alpha=\frac{1}{6} \\
& \text { (bidisperse four-ellipses, } \Sigma_{A P_{\max }} \geqslant 1 / 2 \text { ). }
\end{aligned}
$$

Furthermore, the same cubic dependence (27) as for disks is observed for other values of $\varphi_{M}$, and the data for four-ellipses are very well represented by (25), (27), and (28), within \pm 0.02 with a rms deviation equal to 0.009 .

\section{Power law size distributions}

The data for networks with power law distributions of fracture size can be analyzed in the same way in terms of $\Sigma_{A P}$, which is defined by (9)

$$
\Sigma_{A P}^{2}=\frac{(4-a)^{2}}{(1-a)(7-a)} \frac{\left(\tilde{R}^{7-a}-1\right)\left(\tilde{R}^{1-a}-1\right)}{\left(\tilde{R}^{4-a}-1\right)^{2}} .
$$

When the range of sizes $\tilde{R}$ is fixed, $\Sigma_{A P}$ is maximum for $a=4$, with

$$
\Sigma_{A P_{\max }}^{2}(\tilde{R})=\frac{1}{\tilde{R}^{3}}\left[\frac{\tilde{R}^{3}-1}{3 \ln \tilde{R}}\right]^{2}
$$

Note that in this case, the maximum of $\Sigma_{A P}$ does not correspond to a constant $\varphi(R) R^{3}$ (i.e., $a=3$ ), as it does with $\Phi_{M}=\Phi_{m}$ in bidisperse networks.

The numerical data are displayed in Fig. 7, which is a counterpart of Fig. 6 and many common features with the bidisperse case are observed. Again, for any fixed $\tilde{R}$, the maximum increment is reached for $\Sigma_{A P_{\max }}$ with $a=4$, and $\delta \rho_{3 c_{\max }}^{\prime}$ is well described by (26) with a modified $\alpha$ [Fig. 7(a)],

$$
\begin{aligned}
& \delta \rho_{3 c_{\max }}^{\prime}= \alpha\left[\Sigma_{A P_{\max }}^{1 / 2}-\left(\frac{1}{2}\right)^{1 / 2}\right] \\
& \text { with }\left\{\begin{array}{l}
\left.\alpha=\frac{1}{5} \text { (power law, disks, } \Sigma_{A P_{\max }} \geqslant \frac{1}{2}\right) \\
\left.\alpha=\frac{1}{8} \text { (power law, 4-ellipses, } \Sigma_{A P_{\max }} \geqslant \frac{1}{2}\right)
\end{array}\right.
\end{aligned}
$$

The data for regular polygons are again found very close to that for disks. Elongated four-ellipses always yield smaller increments, and the results for four-rectangles and fourlozenges (with $a=4$ and $\tilde{R}=8$ ) are very close to that for four-ellipses. The variations of $\delta \rho_{3 c_{\max }}^{\prime}$ as functions of $\Sigma_{A P_{\max }}$ and the models (26), (28), and (31) are summarized in Fig. 8(a) for disks and four-ellipses, with bidisperse and power law size distributions. The overall rms deviation of the models from the numerical data for $\Sigma_{A P_{\max }}$ is equal to 0.011 .

Figure 7(b) shows that for $\Sigma_{A P}<\Sigma_{A P_{\max }}$ (i.e., $a \neq 4$ ), $\delta \rho_{3 c}^{\prime}$ can be estimated by a linear interpolation in terms of $\Sigma_{A P}^{3 / 2}$. Thus, a counterpart of (27) applies for power law distributions, with a different exponent,

$$
\delta \rho_{3 c}^{\prime}=\delta \rho_{3 c_{\max }}^{\prime}(\tilde{R})\left[\frac{\Sigma_{A P}}{\Sigma_{A P_{\max }}(\tilde{R})}\right]^{3 / 2} .
$$

The model resulting from the combination of (30)-(32) approximates all the numerical data for disks with a rms deviation equal to 0.012 . Since the results for regular polygons are very close to those for disks, they are very well described by the same model with a rms deviation equal to 0.013. Finally, the rms deviation of the model from the data for ellipses is equal to 0.008 .

\section{Discussion}

Very successful models for $\delta \rho_{3 c}^{\prime}$ have been devised for the investigated size distributions, in the form of (27) or (32), combined with (26), (28), or (31).

It should be noted that they apply only for $\Sigma_{A P_{\max }} \geqslant 1 / 2$. However, the increment is very small for $\Sigma_{A P} \lesssim 1$, well within the error bar \pm 0.05 of (18), and it can be ignored. This explains that the need for a correction was not detected in [5], where combinations of large enough $\tilde{R}$ and $a$ were not explored. It also explains the success of (18) for the mixtures considered in Sec. IV. The quadrilaterals have a wide range of sizes, but $\Sigma_{A P}$ is only 0.75 (Table IX in the Supplemental Material [7]). The fractures in the binary mixtures have sometimes very different areas or bounding radii, but $\Sigma_{A P}$ exceeds 1 only in rare occurrences [see Eqs. (19) and (20) and Tables II and III in the Supplemental Material [7]]. The largest value $\Sigma_{A P}=1.59$ is reached for $10 \%$ of disks mixed with $90 \%$ of eight-ellipses with identical bounding radii. In view of Fig. 6, the increment $\delta \rho_{3 c}^{\prime}$ is expected to remain smaller than 0.1 in mixtures of disks with this value of a $\Sigma_{A P}$ and a similar ratio $(A P)_{M} /(A P)_{m}$.

This is an important observation for practical applications. The criterion $\Sigma_{A P} \lesssim 1$, for which the model (18) applies with good accuracy without need for correction, can be satisfied in a wide range of nontrivial situations, as seen in the above. Furthermore, the simulations conducted in a great variety of polydisperse cases show that the correction $\delta \rho_{3 c}^{\prime}$ remains 


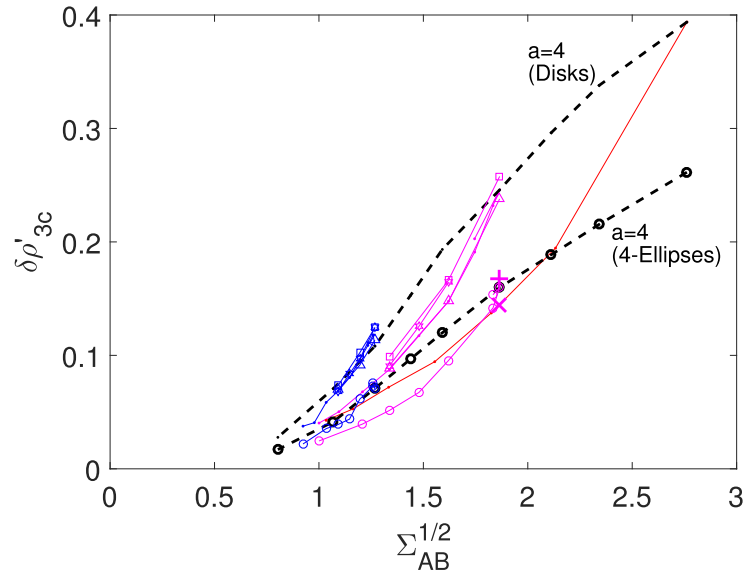

(a)

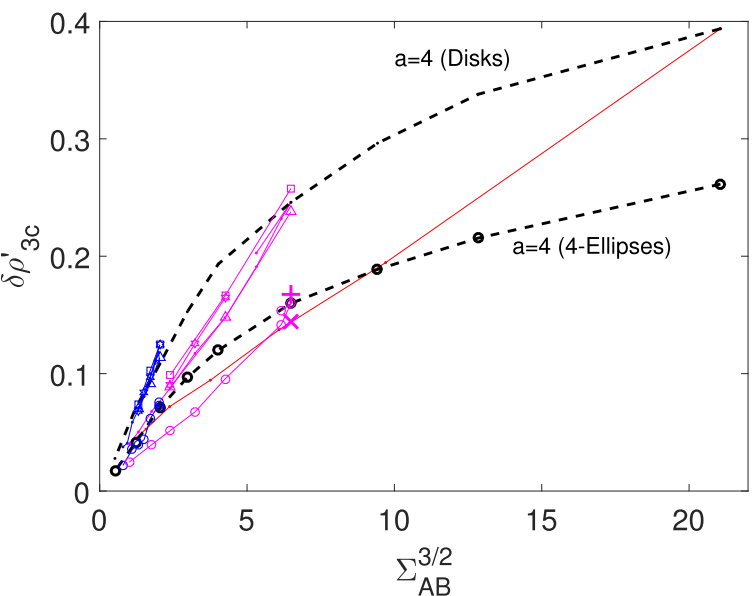

(b)

FIG. 7. The increment $\delta \rho_{3 c}^{\prime}$ in networks with power law size distribution as a function of $\Sigma_{A P}^{1 / 2}$ (a) and of $\Sigma_{A P}^{3 / 2}$ (b). Data are for disks (dots), four-ellipses $(\circ)$, hexagons $(\bowtie)$, squares $(\square)$, triangles $(\triangle)$, four-rectangles $(+)$, and four-lozenges $(\times)$. Colors correspond to $\tilde{R}=4$ (blue), 8 (magenta), and 16 (red). Black broken lines correspond to $a=4$.

smaller than $10 \%$ of the prediction of (18) when $\Sigma_{A P} \lesssim 4$, and smaller than $20 \%$ of the prediction of (18) when $\Sigma_{A P} \lesssim 10$. For illustration, a power law size distribution with exponent $a=2$ and $\tilde{R}=100$ yields $\Sigma_{A P} \approx 9$. Finally, $\delta \rho_{3 c}^{\prime}$ depends on the fracture shape and on the exact form of the size distribution, but the largest increment for any specified value of $\Sigma_{A P}$ corresponds to the bidisperse mixture of disks. Therefore, (26) provides an upper bound for the necessary correction to (18).

Thus, the analyses in Secs. V B and V C have identified the relevant parameter $\Sigma_{A P}$, which provides a quantitative way to assess whether a correction to (18) is necessary and which governs this correction if it is significant. Very successful models for $\delta \rho_{3 c}^{\prime}$ for bidisperse and power law distributions have also been formulated.

Still, even though this is a gratifying and useful step, it is not fully satisfying. Although (27) and (32) have identical forms, the difference of the exponents is unexplained, as

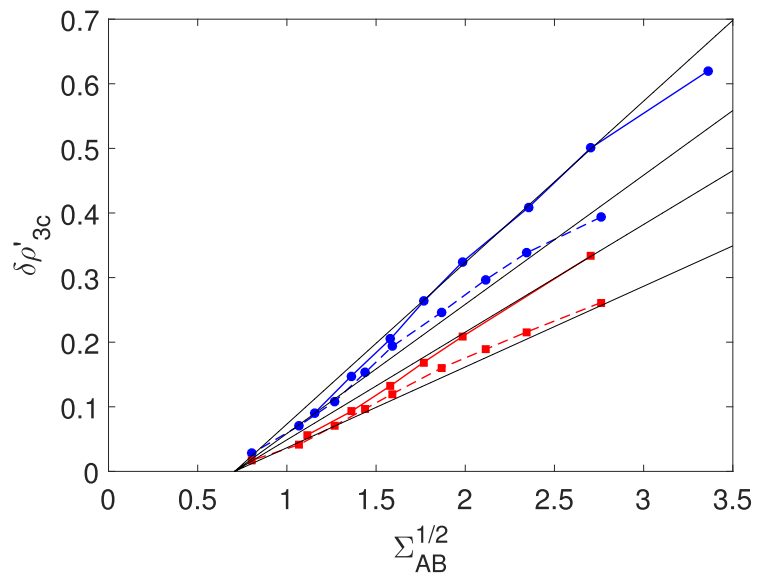

(a) is the change of $\alpha$ in (26), (28), and (31) according to the form of the size distribution. The intrinsic parameter $\Sigma_{A P}$, which is a combination of moments of the fracture geometrical characteristics, defined without reference to the model parameters $\left(\tilde{R}, \varphi_{M}, a\right)$ and therefore applicable a priori to any kind of network, has obviously a high degree of pertinence. But it does not capture by itself all the effects of polydispersity. It probably should be corrected or completed by another, yet unidentified, parameter to provide a unified, model-independant model for $\delta \rho_{3 c}^{\prime}$.

A hint for further analysis might be found in the logarithmic plot of $\delta \rho_{3 c}^{\prime}$ vs $\Sigma_{A P}$ in Fig. 8(b). A transition takes place at $\Sigma_{A P} \sim 2$ between two growth regimes according to $\Sigma_{A P}^{3 / 2}$ and $\Sigma_{A P}^{1 / 2}$. This is probably the reason for the apparent behavior (26) with an offset value, when the small increments in the low $\Sigma_{A P}$ range are disregarded. It probably results from the competition of different combinations of moments that prevail in different ranges of size contrasts.

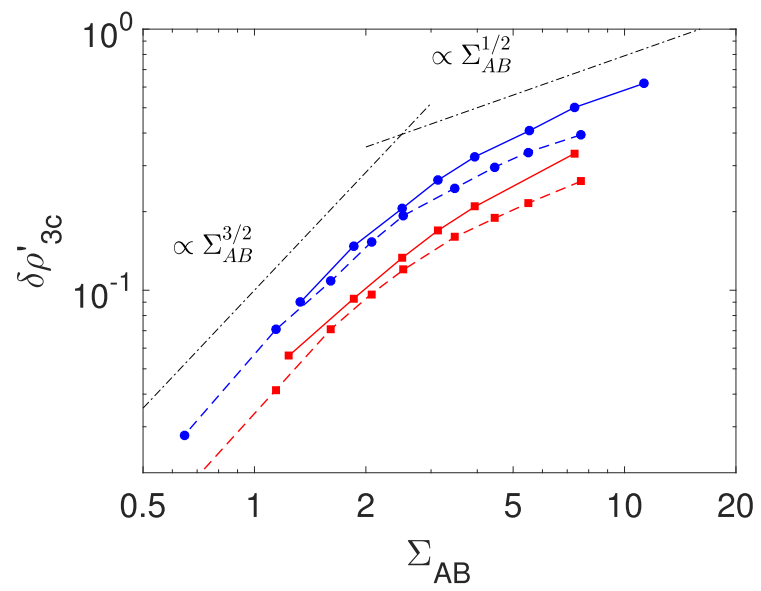

(b)

FIG. 8. The increment $\delta \rho_{3 c}^{\prime}$ in networks of disks (•) or four-ellipses with bidisperse $\left(\varphi_{M}=\varphi_{M_{\max }}\right.$, solid lines) or power law $(a=4$, broken lines) distribution of fracture sizes, as a function of $\Sigma_{A P}^{1 / 2}$ (a) and $\Sigma_{A P}$ (b). The straight lines in (a) correspond to (26). The dash lines in (b) illustrate trends proportional to $\Sigma_{A P}^{3 / 2}$ and $\Sigma_{A P}^{1 / 2}$. 
Finally, the difference in $\alpha$ between regular and elongated fracture shapes is also unexplained, and insufficiently documented at this stage to elaborate.

\section{DISCUSSION OF A HEURISTIC PREDICTIVE MODEI}

\section{A. Principle of the argument}

A heuristic argument was proposed in [6] to predict the percolation thresholds in continuum systems. The average "bonding distance" $l$ is defined as the mean distance between connected objects, and expressed in the form

$$
l^{2}=\frac{1}{V_{\mathrm{ex}}} \int_{V_{\mathrm{ex}}} r^{2} d^{3} \boldsymbol{r} .
$$

Note that $l$ does not depend on the density of objects. It is then postulated that percolation occurs when the average distance between objects with at least two neighbors (of five neighbors in two-dimensional settings, which are not considered here) is smaller than $2 l$, i.e., when the mean number of such objects in a volume $V_{l}=4 \pi l^{3} / 3$ is at least 1 .

Since the number of connections to a given object obeys a Poisson distribution with average $\rho^{\prime}$, the density $\rho_{2}$ of objects with at least two neighbors is given by

$$
\rho_{2}=\rho\left[1-\left(1+\rho^{\prime}\right) e^{-\rho^{\prime}}\right] .
$$

Thus, the criterion $\rho_{2} V_{l} \geqslant 1$ for percolation yields a transcendental equation for the critical concentration $\rho_{c}^{\prime}$ which can be easily solved numerically,

$$
\rho_{c}^{\prime}\left[1-e^{-\rho_{c}^{\prime}}\left(1+\rho_{c}^{\prime}\right)\right]=\frac{V_{\mathrm{ex}}}{V_{l}} .
$$

Although the argument is not substantiated, quite successful examples of application are provided by [6]; it predicts percolation thresholds $\rho_{c}^{\prime}=2.796$ for penetrable spheres and 2.604 for aligned penetrable cubes, in very good agreement with the results 2.735 [29] and 2.598 [30] from direct simulations.

However, a major shortcoming has to be overcome for applications to other situations. The definition (33) of the bonding distance makes sense for the provided examples, with spheres or aligned objects, because an object $F_{2}$ intersects a reference object $F_{1}$ if and only if it is centered within a volume homothetic to that of $F_{1}$, dilated by a factor of 2 . This volume can be identified with $V_{\mathrm{ex}}$, and the integration in (33) can be performed to evaluate its gyration radius $l$.

This does not apply for aspherical, randomly oriented objects. Objects $F_{1}$ and $F_{2}$ centered at $\boldsymbol{r}_{1}$ and $\boldsymbol{r}_{2}$ can intersect or not depending on their relative orientation. An intersection probability $\Pi\left(\boldsymbol{r}_{2}-\boldsymbol{r}_{1}\right)$ can only be defined in average over the orientation. No definite geometrical representation can be given to the excluded volume, and a different definition of $l$ has to be devised. In addition, nothing tells a priori that the second order moment (33) is the most relevant one. Therefore, we introduce the $p$-order moment $l^{(p)}$ of the center-to-center distance of intersecting objects as

$$
l^{(p)}=\frac{\int r^{p} \Pi(\boldsymbol{r}) d^{3} \boldsymbol{r}}{\int \Pi(\boldsymbol{r}) d^{3} \boldsymbol{r}},
$$

where the integrals extend over the whole space. Note that the denominator is equal to $V_{\mathrm{ex}}$.

\section{B. Determination of the moments $\boldsymbol{l}^{(p)}$}

Although cumbersome, the analytic integration of (36) is possible when $p=2$. The final result for two objects $F_{1}$ and $F_{2}$ reads

$$
l_{1,2}^{(2)}=\frac{A_{1} P_{2}\left(R_{g 1}^{2}+R_{h 2}^{2}\right)+A_{2} P_{1}\left(R_{g 2}^{2}+R_{h 1}^{2}\right)}{A_{1} P_{2}+A_{2} P_{1}},
$$

where $A_{i}$ and $P_{i}$ are the object's surface areas and perimeters. $R_{\mathrm{gi}}$ and $R_{\mathrm{hi}}$ are the gyration radii of the object $F_{i}$ and of its contour $\partial F_{i}$,

$$
R_{\mathrm{gi}}^{2}=\frac{1}{A_{i}} \int_{F_{i}} r^{2} d s, \quad R_{\mathrm{hi}}^{2}=\frac{1}{P_{i}} \int_{\partial F_{i}} r^{2} d l .
$$

Formulas for these geometrical characteristics are provided in Table I for various kinds of object shapes. Note that the result (37) applies when the distance $r$ in (36) is taken between the object barycenters. When the two objects are identical, (37) reduces to

$$
l^{(2)}=R_{g}^{2}+R_{h}^{2} .
$$

A global average of the bonding distance $l^{(2)}$ in a network containing objects of various shapes and/or sizes can be obtained by weighting $l_{1,2}^{(2)}$ by the proportion of intersections between $F_{1}$ and $F_{2}$ objects,

$$
\left\langle l^{(2)}\right\rangle=\frac{\iint l_{1,2}^{(2)} V_{\mathrm{ex}, i j} d \rho_{i} d \rho_{j}}{\iint V_{\mathrm{ex}, i j} d \rho_{i} d \rho_{j}}=\frac{\left\langle A R_{g}^{2}\right\rangle}{\langle A\rangle}+\frac{\left\langle P R_{h}^{2}\right\rangle}{\langle P\rangle} .
$$

Analytical integration for $p \neq 2$ is not possible but the moment $l^{(p)}$ can be calculated by numerical Monte Carlo integration. An object $F_{1}$ is placed at the origin, and another object $F_{2}$ is placed at a random position $\boldsymbol{r}$ with a random orientation. The quantities $r^{p}$ are averaged over all the cases when $F_{2}$ intersects $F_{1}$. This was done for objects with a variety of plane convex shapes. The averages were taken over large numbers of intersecting pairs (tens of millions for polygonal shapes and hundreds of millions for disks and ellipses).

The full results for $l^{(1)}$ and $l^{(2)}$ are given in Table $\mathrm{X}$ in the Supplemental Material [7]. In all cases, the measured $l^{(2)}$ agrees within $\pm 10^{-4}$ with (37). Furthermore, a very good approximation of the data for $l^{(1)}$ is provided by the model

$$
l^{(1)} \approx\left[\frac{8 l^{(2)}}{9}\right]^{1 / 2} .
$$

This deviates from the measured data by less than $0.5 \%$ for disks, regular polygons, and moderately elongated objects $(f \leqslant 2)$, and for pairs of such objects, even when they have very different sizes. The deviation increases for very slender objects, but reaches only about $+3 \%$ for ellipses, rectangles, or lozenges with $f=8$. Hence, the combination of (37) and (41) 
TABLE II. The percolation threshold $\rho_{c}^{\prime}$ determined from the numerical simulations and by applying (35) with $V_{l}$ estimated with $l=l^{(1)}$ or $l=\sqrt{l^{(2)}}$, for monodisperse networks of regular or moderately elongated fractures. Numbers in parentheses are the deviations from the simulation results.

\begin{tabular}{lcccc}
\hline \hline \multirow{2}{*}{ Fracture shape } & \multicolumn{2}{l}{ Threshold $\rho_{c}^{\prime}$ determined from } & & \\
Simulations & $(35)$ with $\sqrt{l^{(2)}}$ & $(35)$ with $l^{(1)}$ & $(35)$ with $\sqrt{8 l^{(2)} / 9}$ \\
\hline Disks & 2.303 & $2.083(-0.221)$ & $2.278(-0.026)$ & $2.293(-0.010)$ \\
20-gons & 2.307 & $2.087(-0.220)$ & $2.282(-0.025)$ & $2.298(-0.009)$ \\
Hexagons & 2.331 & $2.125(-0.207)$ & $2.320(-0.011)$ & $2.341(0.010)$ \\
Squares & 2.340 & $2.141(-0.199)$ & $2.339(-0.000)$ & $2.360(0.021)$ \\
Triangles & 2.267 & $2.046(-0.221)$ & $2.250(-0.017)$ & $2.251(-0.016)$ \\
Ellipses $(f=1.5)$ & 2.272 & $2.027(-0.245)$ & $2.227(-0.045)$ & $2.229(-0.043)$ \\
Ellipses $(f=2)$ & 2.220 & $1.926(-0.294)$ & $2.227(-0.089)$ & $2.113(-0.107)$ \\
Rectangles $(f=2)$ & 2.240 & $1.955(-0.286)$ & $2.159(-0.082)$ & $2.146(-0.094)$ \\
Lozenges $(f=2)$ & 2.207 & $1.904(-0.303)$ & $2.111(-0.097)$ & $2.088(-0.119)$ \\
\hline \hline
\end{tabular}

provides a convenient and fairly accurate alternative to the direct measurement of $l^{(1)}$ by Monte Carlo integration.

\section{Percolation threshold predictions}

The predictions for the percolation threshold of fracture networks resulting from (35) when $V_{l}$ is estimated from $l=l^{(1)}$ or $l=\sqrt{l^{(2)}}$ are compared to the results from the numerical simulations in Table II for monodisperse fractures with circular, regular polygonal, and moderately elongated shapes. Using $l^{(2)}$ as in the original argument of [6] yields very decent predictions, which underestimate the real thresholds by only $0.2-0.3$. However, using instead the first order moment $l^{(1)}$ is much more successful. The error ranges from 0 to -0.03 for regular shapes, and it does not exceed -0.1 for aspect ratios $f \leqslant 2$. Interestingly, (35) accounts for the fact that $\rho_{c}^{\prime}$ for $n$-sided regular polygons $(n \geqslant 4)$ is slightly larger than for disks, whereas it is smaller for all other shapes. Finally, $l^{(1)}$ as deduced from (37) and the approximation (41) yields predictions as accurate as the measured value of $l^{(1)}$.

The same success is observed when two of these shapes are mixed in the fracture networks. Mixtures of hexagons and triangles (same bounding radius) or of 20-gons with squares (same bounding radius or same area) in various proportions $(25 / 75 \%, 50 / 50 \%$, and $75 / 25 \%)$ have been considered. The bonding distance $l^{(1)}$ was averaged with the same weighting as for $l^{(2)}$ in (40). Again, the predictions of (35) underestimate the numerical results by at most -0.02 .

However, the argument is much less successful for very elongated shapes. The predicted thresholds are compared in Fig. 9 to the results of the numerical simulations, for ellipses, rectangles, and lozenges with $f=1-16$. The data are plotted as functions of the shape factor $\tilde{\eta}_{g}$ for an easier comparison with the discussion in Secs. III and IV. $V_{l}$ in (35) is based on $l=l^{(1)}$ evaluated from (37) and (41) for convenience, but very similar results are obtained if the values of $l^{(1)}$ from the Monte Carlo integration are used in place of this model. It appears that the prediction (35) fails when the fracture shape strongly departs from circularity. Using $l=\sqrt{l^{(2)}}$ rather than $l^{(1)}$ yields to even larger deviations from the numerical results.

Networks of fractures with polydisperse sizes have also been considered. Only the simplest case of bidisperse disks is discussed in detail here. The predictions of (35) with $l=l^{(1)}$ are compared in Fig. 10(a) to the numerical simulations when the size ratio $\tilde{R}$ varies for various values of the fraction $\varphi_{M}$. Conversely, $\tilde{R}$ is kept constant and the composition quantified by $\Phi_{M}$ [see Eq. (22)] varies in Fig. 10(b). Note that the percolation threshold is expressed in terms of the density $\rho_{3 c}^{\prime}$, which is more appropriate than $\rho_{c}^{\prime}$ for polydisperse networks. While an increase of $\rho_{3 c}^{\prime}$ due to the polydispersity is indeed predicted, its magnitude and its dependence on the size distribution are not correctly captured. In particular, the predictions fail to converge to $\rho_{c}^{\prime}$ for monodisperse networks when $\tilde{R}$ increases while $\varphi_{M}$ is kept constant. The comparison is no more successful if $l^{(1)}$ is replaced by $\sqrt{l^{(2)}}$ or if continuous size distributions are considered. For instance, the increase of $\rho_{3 c}^{\prime}$ over $\rho_{c}^{\prime}$ for monodisperse networks is strongly underestimated for power law distributions of the fracture sizes, whatever the exponent and the ratio of the upper and lower cutoff sizes.

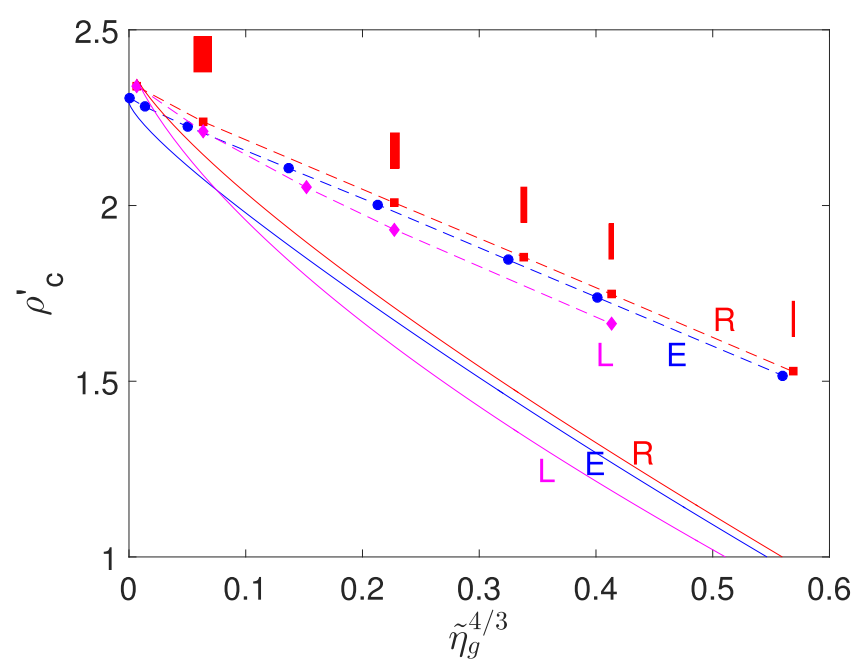

FIG. 9. The percolation thresholds $\rho_{c}^{\prime}$ determined from the numerical simulations (symbols, broken lines) and by applying (35) with $V_{l}$ estimated with $l=l^{(1)}$ as given by (37) and (41) (solid lines), as functions of the shape factor $\tilde{\eta}_{g}$. Data are for networks of ellipses (label E, blue), rectangles (label R, red) or lozenges (label $\mathrm{L}$, magenta). The icons illustrate the shapes of the rectangles for $f=2,4,6,8$, and 16 . 


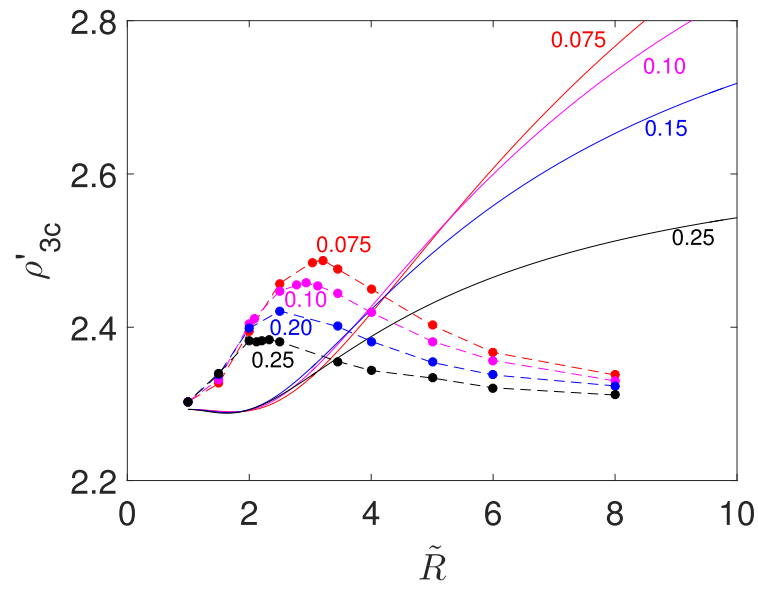

(a)

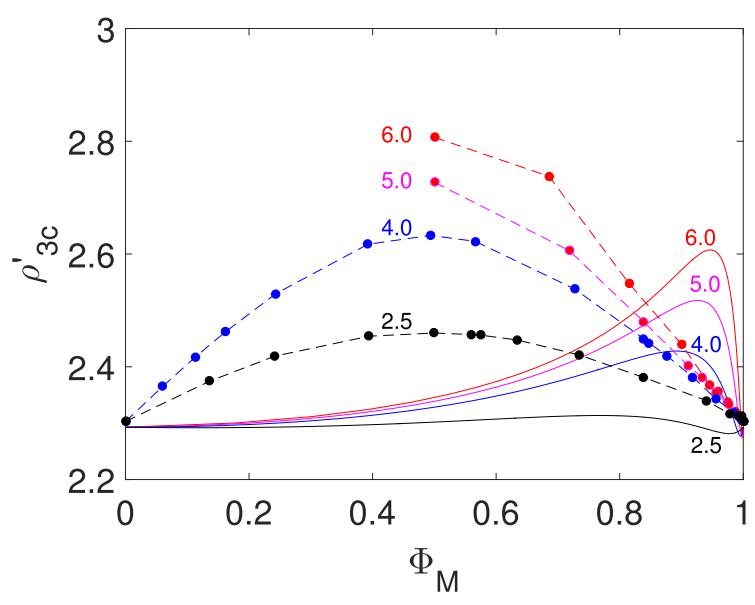

(b)

FIG. 10. The percolation thresholds $\rho_{c}^{\prime}$ determined from the numerical simulations (symbols, broken lines) and by applying (35) with $V_{l}$ estimated with $l=l^{(1)}$ as given by (37) and (41) (solid lines), for bidisperse networks of disks with radii $R_{M}$ and $R_{m}$ in proportions $\varphi_{M}$ and $\varphi_{m}=1-\varphi_{M}$. The data are plotted as functions of $\tilde{R}=R_{M} / R_{m}$ in (a) for various values of the fraction $\varphi_{M}$, indicated next to the curves. The data are plotted as functions of the composition parameter $\Phi_{M}$ in (b) for various ratios $\tilde{R}$, indicated next to the curves.

Finally, the argument also fails for the networks or random quadrilaterals, which is not surprising since they mix fractures with very different and possibly very elongated shapes, with a broad size distribution. The measured threshold $\rho_{3 c}^{\prime}=2.15$ is overestimated by 0.27 when (35) is applied with the value of $l^{(1)}$ determined by Monte Carlo integration.

In summary, the general argument of [6] is very successful and indisputably captures part of the shape dependence of $\rho_{c}^{\prime}$, when appropriately adapted, in networks of fractures of comparable sizes and with shapes that do not strongly depart from circularity. In particular, it accounts for the differences between continuum percolation systems of solid (spheres, cubes) or flat penetrable objects, although the reason why $l^{(2)}$ or $l^{(1)}$ should be used depending on the situation is not elucidated. The account for the influence of the fracture shape is also interesting, although it applies in a limited range where the approximation $\rho_{3 c}^{\prime} \approx 2.3 \pm 0.1$ is probably sufficient for most practical applications.

However, the argument fails to describe the case of elongated fracture shapes. The quantity $\left(V_{l} / V_{\mathrm{ex}}\right)^{1 / 3}$ which determines the right-hand side term in (35) was called the "pointedness" of the object in [6]. It was regarded as a measure of the object's departure from sphericity and was expected to account for the longer average distance between overlapping objects when they are elongated and for its impact on the critical density. Figure 9 shows that it does not succeed in this task. Furthermore, the argument does not succeed either in mixtures of objects with different sizes.

\section{CONCLUDING REMARKS}

The main result in this paper is the model (18) for the dimensionless percolation density $\rho_{3 c}^{\prime}$, which applies with very good accuracy to monodisperse and moderately polydisperse fracture networks. The polydispersity can involve variable fracture shapes and/or sizes. The critical density is unified by the use of the reference volume $\langle A P\rangle$ in the definition of $\rho_{3 c}^{\prime}$, and the shape factor $\left\langle\tilde{\eta}_{g}\right\rangle$ describes its decrease when the fracture shapes are very irregular.

The degree of polydispersity can be quantified by the reduced standard deviation $\Sigma_{A P}$ of $A P$, and the model (18) applies without need for a correction when $\Sigma_{A P} \leqslant 1$. Conversely, strong polydispersity with $\Sigma_{A P}>1$ resulting from broad size distributions increases $\rho_{3 c}^{\prime}$. Its increment depends on the particular form of the size distribution and on the fracture shape in a way that is not yet fully elucidated. However, it does not change the order of magnitude of the critical density, and an upper bound is provided by the increment (26) for bidisperse disks with the same $\Sigma_{A P}$.

For practical applications, the knowledge of several quantities is required to assess the percolating status of a fracture network, namely $\rho,\langle A P\rangle,\left\langle\tilde{\eta}_{g}\right\rangle$, and $\Sigma_{A P}$. Their determination by stereological analysis of two-dimensional trace maps is currently being investigated. A robust procedure that provides reasonable estimates, at least when polydispersivity is moderate, has been devised. For instance, the analysis of a trace map in a plane intersecting a network of random quadrilaterals yields a value of $\rho_{3 c}^{\prime}$ which deviates from the actual one by only $20 \%$, and $\left\langle\tilde{\eta}_{g}\right\rangle$ is obtained with an error of only -0.006 . A full account of these developments will be provided in a separate communication in the near future.

Finally, it should be emphasized that this work can be extended in many ways. For instance, many real fracture networks display a hierarchical character which is due to the fact that different families of fractures are produced at different geological times. Therefore, the newly generated fractures tend to be stopped by the preexisting ones as proposed by [31]. Another important feature is that fractures do not follow a Poisson distribution; in a first approximation this could be accounted for by variations in the local density which could be described by a fractal correlation. Totally different processes were followed by [32] who built fractal networks based on Levy flight processes, and by [33] who applied randomized iteration functions to generate hierarchical fractal networks with adjustable box dimension. 
[1] B. Faybishenko, P. A. Witherspoon, and J. Gale, Dynamics of Fluids And Transport in Fractured Rock, AGU, Geophysical Monograph Series (American Geophysical Union, Washington, DC, 2005), Vol. 162.

[2] E. Charlaix, E. Guyon, and N. River, Solid State Commun. 50, 999 (1984).

[3] I. Balberg, Phys. Rev. B 31, 4053 (1985).

[4] O. Huseby, J.-F. Thovert, and P. M. Adler, J. Phys. A 30, 1415 (1997).

[5] V. V. Mourzenko, J.-F. Thovert, and P. M. Adler, Phys. Rev. E 72, 036103 (2005).

[6] U. Alon, I. Balberg, and A. Drory, Phys. Rev. Lett. 66, 2879 (1991).

[7] See Supplemental Material at http://link.aps.org/supplemental/ 10.1103/PhysRevE.95.042112 for the comprehensive set of numerical data, details about the random quadrilaterals, and auxilliary data pertaining to Sec. VI.

[8] S. Sisavath, V. V. Mourzenko, P. Genthon, J.-F. Thovert, and P. M. Adler, Geophys. J. Int. 157, 917 (2004).

[9] V. V. Mourzenko, J.-F. Thovert, and P. M. Adler, in Proceedings of the International Conference on Rock Joints and Jointed Rock Masses, Tucson, 2009, edited by Pinnaduwa H. S. W. Kulatilake (University of Arizona, Tucson, 2009).

[10] V. V. Mourzenko, J. F. Thovert, and P. M. Adler, Phys. Rev. E 86, 026312 (2012).

[11] G. Lamé, Examen des Différentes Méthodes Employées pour Résoudre les Problèmes de Géométrie (Vve Courcier, Paris, 1818).

[12] P. M. Adler, J.-F. Thovert, and V. V. Mourzenko, Fractured Porous Media (Oxford University Press, Oxford, 2012).

[13] P. M. Adler and J.-F. Thovert, Fractures and Fracture Networks (Kluwer, Dordrecht, 1999).

[14] M. Sahimi, Flow and Transport in Porous Media and Fractured Rock (VCH, New York, 1995).
[15] D. Stauffer and A. Aharony, Introduction to Percolation Theory (Taylor \& Francis, Bristol, PA, 1992).

[16] N. A. M. Araújo, P. Grassberger, B. Kahng, K. J. Schrenk, and R. M. Ziff, Eur. Phys. J.: Spec. Top. 223, 2307 (2014).

[17] I. Balberg, Continuum percolation, in Encyclopedia of Complexity and Systems Science, edited by R. A. Meyers (Springer, New York, 2009).

[18] I. Balberg, C. H. Anderson, S. Alexander, and N. Wagner, Phys. Rev. B 30, 3933 (1984).

[19] A. Isihara, J. Chem. Phys. 18, 1446 (1950).

[20] N. Koudina, R. Gonzalez Garcia, J.-F. Thovert, and P. M. Adler, Phys. Rev. E 57, 4466 (1998).

[21] V. V. Mourzenko, J.-F. Thovert, and P. M. Adler, Phys. Rev. E 84, 036307 (2011).

[22] R. Rosenzweig, V. V. Mourzenko, J.-F. Thovert, and P. M. Adler, Phys. Rev. E 90, 022407 (2014).

[23] V. V. Mourzenko, J.-F. Thovert, and P. M. Adler, Phys. Rev. E 69, 066307 (2004).

[24] E. J. Garboczi, K. A. Snyder, J. F. Douglas, and M. F. Thorpe, Phys. Rev. E 52, 819 (1995).

[25] R. Florian and Z. Neda, arXiv:cond-mat/0110067v1.

[26] M. O. Saar and M. Manga, Phys. Rev. E 65, 056131 (2002).

[27] J. R. de Dreuzy, P. Davey, and O. Bour, Phys. Rev. E 62, 5948 (2000).

[28] Y. B. Yi and E. Tawerghi, Phys. Rev. E 79, 041134 (2009).

[29] C. D. Lorenz and R. M. Ziff, J. Chem. Phys. 114, 3659 (2001).

[30] D. R. Baker, G. Paul, S. Sreenivasan, and H. E. Stanley, Phys. Rev. E 66, 046136 (2002).

[31] F. Conrad and C. Jacquin, Rev. IFP 28, 843 (1973).

[32] T. Clemo and L. Smith, Water Resours. Res. 33, 1763 (1997).

[33] J. A. Acuna and Y. C. Yortsos, Water Resours. Res. 31, 527 (1995). 


\title{
SUPPLEMENTAL MATERIAL
}

\author{
for the article
}

\section{Percolation in three-dimensional fracture networks for arbitrary size and shape distributions}

\author{
by \\ J.-F. Thovert ${ }^{1}$, V.V. Mourzenko ${ }^{2}$ and P.M. Adler ${ }^{3}$
}

\footnotetext{
${ }^{1}$ Institut P'-CNRS, SP2MI, BP 30179, 86962 Futuroscope Chasseneuil, France (thovert@ensma.fr).

2 Institut P'-CNRS, SP2MI, BP 30179, 86962 Futuroscope Chasseneuil, France (murzenko@ensma.fr).

${ }^{3}$ UPMC METIS, BP 105, 4 place Jussieu, 75252 Paris cedex 05, France (pierre.adler@upmc.fr).
} 


\section{DETAILED RESULTS}

The numerical determinations of the percolation thresholds $\rho_{c}^{\prime}$ (for monodispere networks) and $\rho_{3 c}^{\prime}$ (for polydisperse networks) are provided here for:

- Networks of identical fractures in Table I;

- Binary mixtures of 20-gons and rectangles with various aspect ratios and of disks and ellipses with various aspect ratios in Table II;

- Ternary mixtures of disks, 3-ellipses and 9 ellipses, and mixtures of random quadrilaterals in Table III;

- Networks of fractures with identical shapes and bidisperse sizes sorted according to the size ratio $\tilde{R}=R_{M} / R_{m}$ in Table IV or to the fraction $\varphi_{M}$ of large objects in Tables $\mathrm{V}$ and VI;

- Networks of fractures with identical shapes and power law size distributions, sorted according to the fracture size range $\tilde{R}=R_{M} / R_{m}$ in Table VII or to the scaling exponent $a$ in Table VIII.

In addition, some geometrical characteristics of the fracture shapes are reported in Table I, including the shape factor $\tilde{\eta}_{g}$. The average $\left\langle\tilde{\eta}_{g}\right\rangle$ as defined in (Eq. 11) is also provided for the mixtures of shapes in Tables II and III. The polydispersity index $\Sigma_{A P}$ is given for all the polydisperse networks. The size $L_{\max }$ of the largest cells used in the calculations is indicated in all cases, normalized by the largest possible fracture bounding radius $R_{\max }$ for each case.

Unless otherwise stated, the critical density reported in the tables is deduced from the average of the three extrapolated values $\rho_{c}^{(1)}, \rho_{c}^{(x)}$, and $\rho_{c}^{(3)}$ (see Section 2.3 of the article), based on data including calculations for $L$ up to $L_{\max }$, and the uncertainty evaluated by (Eq. 14) is given in parenthesis, multiplied by $10^{3}$. For instance, (12) means \pm 0.012 .

In some cases with power law size distributions (Tables VII and VIII), the extrapolation step was skipped and the reported critical density is $\rho_{L 3 c}^{\prime(1)}$ for $L=L_{M a x}$. These cases are indicated by a mark" $(=)$ " next to the value of $L_{\max }$. 


\begin{tabular}{|c|c|c|c|c|c|c|}
\hline Shape & $A$ & $P$ & $A P / 2$ & $\tilde{\eta}_{g}$ & $L_{\max }$ & $\rho_{c}^{\prime}$ \\
\hline \multicolumn{7}{|c|}{$n$-sided Regular Polygons } \\
\hline$n=3$ & 1.299 & 5.196 & 3.375 & 0.0906 & 80 & $2.270(5)$ \\
\hline$n=4$ & 2.000 & 5.657 & 5.656 & 0.0228 & 80 & $2.338(5)$ \\
\hline$n=6$ & 2.598 & 6.000 & 7.794 & 0.0038 & 80 & $2.334(4)$ \\
\hline$n=20$ & 3.090 & 6.257 & 9.668 & 0.0000 & 80 & $2.303(6)$ \\
\hline \multicolumn{7}{|c|}{ Disks and Ellipses with aspect ratio $f$} \\
\hline$f=1$ & 3.142 & 6.283 & 9.870 & 0.0000 & 160 & $2.303(4)$ \\
\hline$f=1.5$ & 2.094 & 5.289 & 5.538 & 0.0392 & 120 & $2.275(7)$ \\
\hline$f=2$ & 1.571 & 4.844 & 3.805 & 0.1056 & 120 & $2.220(4)$ \\
\hline$f=3$ & 1.047 & 4.455 & 2.333 & 0.2254 & 120 & $2.102(4)$ \\
\hline$f=4$ & 0.785 & 4.289 & 1.684 & 0.3140 & 120 & $1.999_{(3)}$ \\
\hline$f=6$ & 0.524 & 4.150 & 1.087 & 0.4305 & 120 & $1.845(3)$ \\
\hline$f=8$ & 0.393 & 4.093 & 0.804 & 0.5039 & 80 & $1.737(3)$ \\
\hline$f=16$ & 0.196 & 4.029 & 0.396 & 0.6471 & 80 & $1.515(2)$ \\
\hline \multicolumn{7}{|c|}{ Rectangles with aspect ratio $f$} \\
\hline$f=1$ & 2.000 & 5.657 & 5.657 & 0.0228 & 80 & $2.338(5)$ \\
\hline$f=2$ & 1.600 & 5.367 & 4.293 & 0.1260 & 80 & $2.241(3)$ \\
\hline$f=4$ & 0.941 & 4.851 & 2.283 & 0.3296 & 80 & $2.006(4)$ \\
\hline$f=6$ & 0.649 & 4.603 & 1.493 & 0.4435 & 80 & $1.852(3)$ \\
\hline$f=8$ & 0.492 & 4.465 & 1.099 & 0.5152 & 80 & $1.748(4)$ \\
\hline$f=16$ & 0.249 & 4.242 & 0.528 & 0.6552 & 80 & $1.530(4)$ \\
\hline \multicolumn{7}{|c|}{ Lozenges with aspect ratio $f$} \\
\hline$f=1$ & 2.000 & 5.657 & 5.657 & 0.0228 & 80 & $2.338(5)$ \\
\hline$f=2$ & 1.000 & 4.472 & 2.236 & 0.1260 & 40 & $2.202(4)$ \\
\hline$f=3$ & 0.667 & 4.216 & 1.406 & 0.2431 & 40 & $2.049(3)$ \\
\hline$f=4$ & 0.500 & 4.123 & 1.031 & 0.3296 & 40 & $1.929(3)$ \\
\hline$f=8$ & 0.250 & 4.031 & 0.504 & 0.5152 & 40 & $1.664(2)$ \\
\hline
\end{tabular}

TABLE I. Data for monodisperse networks of identical fractures: fracture area $A$, perimeter $P$, excluded volume $A P / 2$, shape factor $\tilde{\eta}_{g}$, largest cell size $L_{\text {max }}$ used in the calculations and percolation threshold $\rho_{c}^{\prime}$. All lengths are normalized by the fracture bounding radius. 


\begin{tabular}{|c|c|c|c|c|c|c|c|c|c|}
\hline \multirow[t]{2}{*}{$f$} & $p_{1}$ & $\left\langle\tilde{\eta}_{g}\right\rangle$ & $\Sigma_{A P}$ & $\frac{L_{\max }}{R_{\max }}$ & $\rho_{3 c}^{\prime}$ & $\left\langle\tilde{\eta}_{g}\right\rangle$ & $\Sigma_{A P}$ & $\frac{L_{\max }}{R_{\max }}$ & $\rho_{3 c}^{\prime}$ \\
\hline & & \multicolumn{4}{|c|}{ Fractures with identical surface areas } & \multicolumn{4}{|c|}{ Fractures with identical bounding radii } \\
\hline & & \multicolumn{8}{|c|}{20 -gons and rectangles with aspect ratio $f$} \\
\hline 1 & 0.00 & 0.0228 & 0.000 & 80.0 & $2.338(5)$ & 0.0228 & 0.000 & 80 & $2.338(5)$ \\
\hline 1 & 0.25 & 0.0200 & 0.049 & 76.1 & $2.328(7)$ & 0.0183 & 0.261 & 80 & $2.325(5)$ \\
\hline 1 & 0.50 & 0.0166 & 0.058 & 108.3 & $2.323(6)$ & 0.0139 & 0.262 & 120 & $2.318(6)$ \\
\hline 1 & 0.75 & 0.0119 & 0.052 & 68.3 & $2.313(10)$ & 0.0092 & 0.009 & 80 & $2.310(6)$ \\
\hline 1 & 1.00 & 0.0000 & 0.000 & 80.0 & $2.303(6)$ & 0.0000 & 0.000 & 80 & $2.303(6)$ \\
\hline 2 & 0.00 & 0.1260 & 0.000 & 80.0 & $2.241(3)$ & 0.1260 & 0.000 & 80 & $2.241(3)$ \\
\hline 2 & 0.25 & 0.1113 & 0.073 & 74.4 & $2.252(3)$ & 0.0963 & 0.413 & 120 & $2.269(5)$ \\
\hline 2 & 0.50 & 0.0929 & 0.088 & 103.2 & $2.268(4)$ & 0.0707 & 0.385 & 160 & $2.285(9)$ \\
\hline 2 & 0.75 & 0.0672 & 0.079 & 63.2 & $2.286(8)$ & 0.0456 & 0.280 & 120 & $2.300(6)$ \\
\hline 2 & 1.00 & 0.0000 & 0.000 & 80.0 & $2.303(6)$ & 0.0000 & 0.000 & 80 & $2.303(6)$ \\
\hline 4 & 0.00 & 0.3296 & 0.000 & 80.0 & $2.006(4)$ & 0.3296 & 0.000 & 80 & $2.006(4)$ \\
\hline 4 & 0.10 & & & & & 0.2782 & 0.733 & 80 & $2.085(4)$ \\
\hline 4 & 0.25 & 0.2964 & 0.134 & 71.0 & $2.050(5)$ & 0.2199 & 0.775 & 80 & $2.159(4)$ \\
\hline 4 & 0.50 & 0.2519 & 0.168 & 62.1 & 2.106 ( 4) & 0.1488 & 0.618 & 80 & 2.229 (9) \\
\hline 4 & 0.75 & 0.1862 & 0.159 & 53.1 & $2.188(6)$ & 0.0907 & 0.409 & 80 & $2.274(4)$ \\
\hline 4 & 1.00 & 0.0000 & 0.000 & 80.0 & $2.303(6)$ & 0.0000 & 0.000 & 80 & $2.303(6)$ \\
\hline 8 & 0.00 & 0.5152 & 0.000 & 80.0 & $1.748(4)$ & 0.5152 & 0.000 & 80 & $1.748(4)$ \\
\hline 8 & 0.10 & & & & & 0.3877 & 1.314 & 40 & 1.927 (6) \\
\hline 8 & 0.25 & 0.4730 & 0.214 & 34.0 & $1.789(6)$ & 0.2793 & 1.145 & 40 & $2.066(5)$ \\
\hline 8 & 0.50 & 0.4126 & 0.283 & 28.0 & $1.861(4)$ & 0.1743 & 0.796 & 40 & $2.190(9)$ \\
\hline 8 & 0.75 & 0.3148 & 0.285 & 43.9 & $2.003(5)$ & 0.1014 & 0.493 & 80 & $2.259(5)$ \\
\hline \multirow[t]{2}{*}{8} & 1.00 & 0.0000 & 0.000 & 80.0 & $2.303(6)$ & 0.0000 & 0.000 & 80 & $2.303(6)$ \\
\hline & & \multicolumn{8}{|c|}{ Disks and ellipses with aspect ratio $f$} \\
\hline 4 & 0.00 & 0.3140 & 0.000 & 120.0 & 1.999 ( 3$)$ & 0.3140 & 0.000 & 120 & $1.999(3)$ \\
\hline 4 & 0.10 & & & & & 0.2554 & 0.981 & 40 & $2.113(6)$ \\
\hline 4 & 0.25 & 0.2815 & 0.124 & 35.0 & 2.041 ( 8) & 0.1946 & 0.950 & 40 & $2.188(13)$ \\
\hline 4 & 0.50 & 0.2386 & 0.154 & 30.0 & 2.105 ( 6) & 0.1265 & 0.708 & 40 & $2.252(7)$ \\
\hline 4 & 0.75 & 0.1756 & 0.145 & 50.0 & $2.187(3)$ & 0.0750 & 0.453 & 40 & $2.285(7)$ \\
\hline 4 & 1.00 & 0.0000 & 0.000 & 160.0 & $2.303(4)$ & 0.0000 & 0.000 & 160 & $2.303(4)$ \\
\hline 8 & 0.00 & 0.5039 & 0.000 & 80.0 & $1.737(3)$ & 0.5039 & 0.000 & 80 & $1.737(3)$ \\
\hline 8 & 0.02 & & & & & 0.4647 & 1.289 & 40 & $1.810(4)$ \\
\hline 8 & 0.10 & & & & & 0.3572 & 1.590 & 40 & 1.959 (6) \\
\hline 8 & 0.25 & 0.4637 & 0.224 & 33.5 & $1.775(3)$ & 0.2471 & 1.279 & 40 & 2.091(13) \\
\hline 8 & 0.50 & 0.4057 & 0.296 & 27.1 & $1.845(5)$ & 0.1492 & 0.849 & 40 & 2.201 (6) \\
\hline 8 & 0.75 & 0.3108 & 0.301 & 61.8 & 1.986 ( 3) & 0.0851 & 0.516 & 40 & $2.263(9)$ \\
\hline 8 & 1.00 & 0.0000 & 0.000 & 160.0 & $2.303(4)$ & 0.0000 & 0.000 & 160 & $2.303(4)$ \\
\hline \multicolumn{6}{|c|}{ Hexagons $(50 \%)+$ Triangles $(50 \%)$} & 0.0505 & 0.396 & 40 & $2.305(7)$ \\
\hline \multicolumn{6}{|c|}{ Hexagons $(50 \%)+4$-Rectangles $(50 \%)$} & 0.1608 & 0.547 & 40 & $2.237(6)$ \\
\hline \multicolumn{6}{|c|}{ Squares $(50 \%)+8$-Rectangles $(50 \%)$} & 0.2161 & 0.675 & 40 & $2.173(5)$ \\
\hline
\end{tabular}

TABLE II. Data for networks containing fractures with two different shapes $\left(p_{1}\right.$ is the fraction of 20-gons or disks): the shape factor $\tilde{\eta}_{g}$, the polydispersity index $\Sigma_{A P}$, the largest cell size $L_{\max }$ and the percolation threshold $\rho_{3 c}^{\prime}$. 


\begin{tabular}{lcccc}
\hline Composition & $\left\langle\tilde{\eta}_{g}\right\rangle$ & $\Sigma_{A P}$ & $\frac{L_{\max }}{R_{\max }}$ & $\rho_{3 c}^{\prime}$ \\
\hline Disks + 3-Ellipses + 9-Ellipses (equal number densities) & 0.1573 & 0.928 & 40 & $2.194(6)$ \\
Disks + 3-Ellipses + 9-Ellipses (equal volumetric areas) & 0.3050 & 1.356 & 40 & $1.976(4)$ \\
Random quadrilaterals & 0.2108 & 0.753 & 40 & $2.148(3)$
\end{tabular}

TABLE III. Data for networks containing fractures with three different shapes or random quadrilaterals: the shape factor $\tilde{\eta}_{g}$, the polydispersity index $\Sigma_{A P}$, the largest cell size $L_{\text {max }}$ and the percolation threshold $\rho_{3 c}^{\prime}$. The disks, 3-ellipses and 9-ellipses have identical bounding radii. 


\begin{tabular}{|c|c|c|c|}
\hline$\varphi_{M}$ & $\Sigma_{A P}$ & $\frac{L_{\max }}{R_{M}}$ & $\rho_{3 c}^{\prime}$ \\
\hline \multicolumn{4}{|c|}{ Disks, $R_{M} / R_{m}=2.5$} \\
\hline 0.000 & 0.000 & 160 & $2.303(4)$ \\
\hline 0.010 & 1.270 & 48.7 & $2.372(8)$ \\
\hline 0.020 & 1.584 & 49.4 & $2.415(8)$ \\
\hline 0.040 & 1.808 & 50.8 & $2.450(6)$ \\
\hline 0.060 & 1.850 & 34.8 & $2.450(8)$ \\
\hline 0.075 & 1.837 & 35.6 & $2.446(12)$ \\
\hline 0.080 & 1.828 & 35.8 & $2.446(11)$ \\
\hline 0.100 & 1.782 & 36.8 & $2.436(10)$ \\
\hline 0.150 & 1.635 & 39.1 & $2.416(6)$ \\
\hline 0.250 & 1.360 & 44.0 & $2.376(6)$ \\
\hline 0.400 & 1.046 & 51.2 & $2.344(7)$ \\
\hline 0.500 & 0.880 & 28.0 & $2.328(18)$ \\
\hline 0.600 & 0.733 & 30.4 & $2.314(9)$ \\
\hline 0.750 & 0.529 & 34.0 & $2.309(7)$ \\
\hline 0.900 & 0.310 & 37.6 & $2.302(15)$ \\
\hline 0.950 & 0.214 & 38.9 & $2.308(11)$ \\
\hline 0.990 & 0.094 & 39.7 & $2.300(9)$ \\
\hline 1.000 & 0.000 & 160 & $2.303(4)$ \\
\hline \multicolumn{4}{|c|}{ Disks, $R_{M} / R_{m}=5$} \\
\hline 0.000 & 0.000 & 160 & $2.303(4)$ \\
\hline 0.005 & 5.399 & 32.6 & $2.695(9)$ \\
\hline 0.0079 & 5.545 & 33.1 & $2.712(11)$ \\
\hline 0.010 & 5.508 & 24.9 & $2.706(13)$ \\
\hline 0.020 & 4.989 & 25.9 & $2.584(22)$ \\
\hline 0.040 & 4.077 & 27.8 & $2.474(6)$ \\
\hline 0.075 & 3.171 & 31.2 & $2.381(16)$ \\
\hline 0.100 & 2.776 & 33.6 & $2.375(8)$ \\
\hline 0.150 & 2.259 & 25.6 & $2.346(14)$ \\
\hline 0.250 & 1.678 & 32.0 & $2.324(10)$ \\
\hline 0.500 & 0.984 & 48.0 & $2.308(6)$ \\
\hline 0.900 & 0.330 & 36.8 & $2.299(8)$ \\
\hline 1.000 & 0.000 & 160 & $2.303(4)$ \\
\hline \multicolumn{4}{|c|}{ 4-Ellipses, $R_{M} / R_{m}=2.5$} \\
\hline 0.000 & 0.000 & 120 & $1.999(3)$ \\
\hline 0.010 & 1.269 & 32.5 & $2.046(3)$ \\
\hline 0.020 & 1.584 & 32.9 & $2.072(3)$ \\
\hline 0.040 & 1.808 & 33.9 & $2.092(4)$ \\
\hline 0.0602 & 1.850 & 34.9 & $2.092(4)$ \\
\hline 0.080 & 1.828 & 35.8 & 2.087 ( 3 ) \\
\hline 0.150 & 1.635 & 39.3 & $2.064(3)$ \\
\hline 0.250 & 1.360 & 22.0 & $2.040(6)$ \\
\hline 0.500 & 0.880 & 28.0 & $2.015(4)$ \\
\hline 1.000 & 0.000 & 120 & $1.999(3)$ \\
\hline
\end{tabular}

\begin{tabular}{lccc}
\hline$\varphi_{M}$ & $\Sigma_{A P}$ & $\frac{L_{\max }}{R_{M}}$ & \multicolumn{1}{c}{$\rho_{3 c}^{\prime}$} \\
\hline Disks, & $R_{M} / R_{m}=3.45$ \\
0.000 & 0.000 & 160 & $2.303(4)$ \\
0.010 & 2.843 & 23.7 & $2.524(11)$ \\
0.020 & 3.111 & 24.3 & $2.568(8)$ \\
0.0238 & 3.124 & 24.5 & $2.566(9)$ \\
0.040 & 3.015 & 25.4 & $2.537(8)$ \\
0.075 & 2.634 & 27.4 & $2.463(10)$ \\
0.100 & 2.400 & 28.8 & $2.435(6)$ \\
0.150 & 2.040 & 31.7 & $2.390(10)$ \\
0.250 & 1.575 & 37.4 & $2.348(8)$ \\
0.500 & 0.952 & 25.8 & $2.304(13)$ \\
0.900 & 0.324 & 37.1 & $2.302(12)$ \\
0.950 & 0.224 & 38.6 & $2.302(9)$ \\
0.990 & 0.098 & 39.7 & $2.299(13)$ \\
1.000 & 0.000 & 160 & $2.303(4)$ \\
\hline Disks, & $R_{M} / R_{m}=6$ & \\
0.000 & 0.000 & 160 & $2.303(4)$ \\
0.0046 & 7.314 & 27.2 & $2.804(11)$ \\
0.010 & 6.791 & 28.0 & $2.684(9)$ \\
0.020 & 5.679 & 29.3 & $2.519(8)$ \\
0.040 & 4.389 & 32.0 & $2.419(6)$ \\
0.075 & 3.307 & 36.7 & $2.356(7)$ \\
0.100 & 2.867 & 30.0 & $2.349(9)$ \\
0.150 & 2.309 & 35.0 & $2.329(12)$ \\
0.250 & 1.700 & 45.0 & $2.316(5)$ \\
1.000 & 0.000 & 160 & $2.303(4)$ \\
\hline & & &
\end{tabular}

\begin{tabular}{lccc}
\hline$\varphi_{M}$ & \multicolumn{4}{c}{$\Sigma_{A P}$} & $\frac{L_{\max }}{R_{M}}$ & $\rho_{3 c}^{\prime}$ \\
\hline Disks, & $R_{M} / R_{m}=4$ \\
0.000 & 0.000 & 160 & $2.303(4)$ \\
0.001 & 1.873 & 30.0 & $2.363(6)$ \\
0.002 & 2.500 & 30.1 & $2.414(4)$ \\
0.003 & 2.898 & 30.2 & $2.461(7)$ \\
0.005 & 3.379 & 30.4 & $2.529(7)$ \\
0.010 & 3.846 & 30.9 & $2.612(11)$ \\
0.015 & 3.937 & 31.3 & $2.627(6)$ \\
0.020 & 3.903 & 31.8 & $2.611(18)$ \\
0.040 & 3.507 & 33.6 & $2.529(6)$ \\
0.075 & 2.898 & 36.7 & $2.456(15)$ \\
0.080 & 2.830 & 24.8 & $2.435(16)$ \\
0.100 & 2.589 & 26.0 & $2.401(12)$ \\
0.150 & 2.153 & 29.0 & $2.369(15)$ \\
0.250 & 1.629 & 35.0 & $2.326(9)$ \\
0.500 & 0.969 & 50.0 & $2.311(9)$ \\
0.750 & 0.565 & 65.0 & $2.306(6)$ \\
0.900 & 0.328 & 74.0 & $2.303(3)$ \\
0.990 & 0.099 & 39.7 & $2.302(7)$ \\
1.000 & 0.000 & 160 & $2.303(4)$ \\
\hline Disks, & $R_{M} / R_{m}=8$ & \\
0.000 & 0.000 & 160 & $2.303(4)$ \\
0.0019 & 11.29 & 20.3 & $2.923(25)$ \\
0.005 & 10.14 & 20.7 & $2.704(29)$ \\
0.010 & 8.321 & 21.4 & $2.537(24)$ \\
0.020 & 6.376 & 34.2 & $2.417(17)$ \\
0.040 & 4.670 & 25.6 & $2.354(19)$ \\
0.075 & 3.423 & 30.5 & $2.328(12)$ \\
0.100 & 2.942 & 25.5 & $2.310(17)$ \\
0.150 & 2.350 & 30.7 & $2.310(7)$ \\
0.250 & 1.719 & 41.2 & $2.304(13)$ \\
1.000 & 0.000 & 160 & $2.303(4)$ \\
\hline & & &
\end{tabular}

\begin{tabular}{lllll}
\hline \multicolumn{4}{l}{ 4-Ellipses, $R_{M} / R_{m}=4$} \\
0.000 & 0.000 & 120 & $1.999(3)$ \\
0.010 & 3.846 & 30.9 & $2.208(5)$ \\
0.0154 & 3.937 & 31.4 & $2.208(6)$ \\
0.020 & 3.903 & 31.8 & $2.200(7)$ \\
0.040 & 3.507 & 22.4 & $2.136(9)$ \\
0.050 & 3.309 & 23.0 & $2.110(14)$ \\
0.080 & 2.830 & 24.8 & $2.074(7)$ \\
0.150 & 2.153 & 29.0 & $2.037(13)$ \\
1.000 & 0.000 & 120 & $1.999(3)$ \\
\hline
\end{tabular}

\begin{tabular}{llll}
\hline \multicolumn{4}{l}{ Squares, $R_{M} / R_{m}=4$} \\
0.000 & 0.000 & 80.0 & $2.338(5)$ \\
0.015 & 3.937 & 20.9 & $2.667(9)$ \\
0.075 & 2.898 & 24.5 & $2.480(10)$ \\
1.000 & 0.000 & 80.0 & $2.338(5)$ \\
\hline \multicolumn{4}{l}{ Triangles, $R_{M} / R_{m}=4$} \\
0.000 & 0.000 & 80.0 & $2.271(8)$ \\
0.015 & 3.937 & 20.9 & $2.576(11)$ \\
0.075 & 2.898 & 24.5 & $2.400(6)$ \\
1.000 & 0.000 & 80.0 & $2.271(8)$ \\
\hline
\end{tabular}

TABLE IV. Data for networks of fractures with identical shapes and bidisperse sizes, with bounding radii $R_{M}$ and $R_{m}$ : fraction $\varphi_{M}$ of large fractures, polydispersity index $\Sigma_{A P}$, largest cell size $L_{\max }$ used in the calculations and percolation threshold $\rho_{3 c}^{\prime}$. 


\begin{tabular}{lllc}
\hline$\tilde{R}$ & $\Sigma_{A P}$ & $\frac{L_{\max }}{R_{M}}$ & $\rho_{3 c}^{\prime}$ \\
\hline Disks, & $\varphi_{M}=0.500$ \\
10.00 & 0.998 & 44.0 & $2.298(11)$ \\
5.000 & 0.984 & 48.0 & $2.308(6)$ \\
4.000 & 0.969 & 50.0 & $2.311(9)$ \\
3.448 & 0.952 & 25.8 & $2.304(13)$ \\
3.333 & 0.947 & 26.0 & $2.316(8)$ \\
2.500 & 0.880 & 28.0 & $2.328(18)$ \\
2.000 & 0.778 & 30.0 & $2.335(5)$ \\
1.667 & 0.645 & 32.0 & $2.328(11)$ \\
1.429 & 0.489 & 34.0 & $2.318(7)$ \\
1.250 & 0.323 & 36.0 & $2.311(8)$ \\
1.111 & 0.157 & 38.0 & $2.299(7)$ \\
1.000 & 0.000 & 160 & $2.303(4)$ \\
\hline Disks, & $\varphi_{M}=0.100$ & \\
8.000 & 2.942 & 25.5 & $2.310(17)$ \\
6.000 & 2.867 & 30.0 & $2.349(9)$ \\
5.000 & 2.776 & 33.6 & $2.375(8)$ \\
4.000 & 2.589 & 26.0 & $2.401(12)$ \\
3.448 & 2.400 & 28.9 & $2.435(6)$ \\
3.125 & 2.241 & 31.0 & $2.445(8)$ \\
2.929 & 2.121 & 32.6 & $2.449(9)$ \\
2.778 & 2.014 & 33.9 & $2.444(11)$ \\
2.500 & 1.782 & 36.8 & $2.436(10)$ \\
2.080 & 1.334 & 21.3 & $2.393(17)$ \\
2.000 & 1.235 & 22.0 & $2.400(14)$ \\
1.500 & 0.576 & 28.0 & $2.326(7)$ \\
1.000 & 0.000 & 160 & $2.303(6)$ \\
\hline
\end{tabular}

\begin{tabular}{lllc}
\hline$\tilde{R}$ & $\Sigma_{A P}$ & $\frac{L_{\max }}{R_{M}}$ & $\rho_{3 c}^{\prime}$ \\
\hline Disks, & $\varphi_{M}=0.250$ \\
8.000 & 1.719 & 41.2 & $2.304(13)$ \\
6.000 & 1.700 & 45.0 & $2.316(5)$ \\
5.000 & 1.678 & 32.0 & $2.324(10)$ \\
4.000 & 1.629 & 35.0 & $2.326(9)$ \\
3.448 & 1.575 & 37.4 & $2.348(8)$ \\
2.500 & 1.360 & 44.0 & $2.376(6)$ \\
2.326 & 1.287 & 45.8 & $2.380(9)$ \\
2.201 & 1.225 & 47.3 & $2.378(4)$ \\
2.128 & 1.184 & 48.2 & $2.376(7)$ \\
2.000 & 1.102 & 25.0 & $2.365(17)$ \\
1.500 & 0.645 & 30.0 & $2.337(8)$ \\
1.000 & 0.000 & 160 & $2.303(4)$ \\
\hline Disks, & $\varphi_{M}=0.075$ & \\
8.000 & 3.423 & 30.5 & $2.328(12)$ \\
6.000 & 3.307 & 36.7 & $2.356(7)$ \\
5.000 & 3.171 & 31.2 & $2.381(16)$ \\
4.000 & 2.898 & 36.7 & $2.456(15)$ \\
3.448 & 2.634 & 27.5 & $2.463(10)$ \\
3.213 & 2.483 & 29.0 & $2.469(19)$ \\
3.030 & 2.346 & 30.4 & $2.471(18)$ \\
2.500 & 1.837 & 35.6 & $2.446(12)$ \\
2.000 & 1.209 & 43.0 & $2.390(8)$ \\
1.500 & 0.531 & 55.3 & $2.320(6)$ \\
1.000 & 0.000 & 160 & $2.303(4)$ \\
\hline & & &
\end{tabular}

\begin{tabular}{lllc}
\hline$\tilde{R}$ & $\Sigma_{A P}$ & $\frac{L_{\max }}{R_{M}}$ & $\rho_{3 c}^{\prime}$ \\
\hline Disks, & $\varphi_{M}=0.150$ \\
8.000 & 2.350 & 30.7 & $2.310(7)$ \\
6.000 & 2.309 & 35.0 & $2.329(12)$ \\
5.000 & 2.259 & 25.6 & $2.346(14)$ \\
4.000 & 2.153 & 29.0 & $2.369(15)$ \\
3.448 & 2.040 & 31.7 & $2.390(10)$ \\
2.500 & 1.635 & 39.2 & $2.416(6)$ \\
2.000 & 1.219 & 23.0 & $2.386(10)$ \\
1.500 & 0.625 & 28.7 & $2.331(11)$ \\
1.000 & 0.000 & 160 & $2.303(4)$ \\
\hline Disks, & $\varphi_{M}=0.040$ & \\
8.000 & 4.670 & 25.6 & $2.354(19)$ \\
6.000 & 4.389 & 32.0 & $2.419(6)$ \\
5.000 & 4.077 & 27.8 & $2.474(6)$ \\
4.000 & 3.507 & 33.6 & $2.529(6)$ \\
3.448 & 3.015 & 25.5 & $2.537(8)$ \\
2.500 & 1.808 & 50.9 & $2.450(6)$ \\
1.000 & 0.000 & 160 & $2.303(4)$ \\
\hline Disks, & $\varphi_{M}=0.020$ & \\
8.000 & 6.376 & 34.2 & $2.417(17)$ \\
6.000 & 5.679 & 29.3 & $2.519(8)$ \\
5.000 & 4.989 & 25.9 & $2.584(22)$ \\
4.000 & 3.903 & 31.8 & $2.611(18)$ \\
3.448 & 3.111 & 24.3 & $2.568(8)$ \\
2.500 & 1.584 & 49.4 & $2.415(8)$ \\
1.000 & 0.000 & 160 & $2.303(4)$ \\
\hline Disks, & $\varphi_{M}=0.010$ & \\
8.000 & 8.321 & 21.4 & $2.537(24)$ \\
6.000 & 6.791 & 28.0 & $2.684(9)$ \\
5.000 & 5.508 & 25.0 & $2.706(13)$ \\
4.000 & 3.846 & 30.9 & $2.612(11)$ \\
3.448 & 2.843 & 23.8 & $2.514(11)$ \\
2.500 & 1.270 & 48.7 & $2.372(8)$ \\
1.000 & 0.000 & 160 & $2.303(4)$ \\
\hline & & &
\end{tabular}

TABLE V. Data for networks of fractures with circular shape and bidisperse sizes, with a fraction $\varphi_{M}$ of large fractures: ratio of the bounding radii $\tilde{R}=R_{M} / R_{m}$, polydispersity index $\Sigma_{A P}$, largest cell size $L_{\max }$ and percolation threshold $\rho_{3 c}^{\prime}$. 


\begin{tabular}{lcccc}
\hline$R_{M} / R_{m}$ & $\varphi_{M}$ & $\Sigma_{A P}$ & $\frac{L_{\max }}{R_{M}}$ & $\rho_{3 c}^{\prime}$ \\
\hline Disks & & & & \\
2.080 & 0.1000 & 1.334 & 21.3 & $2.393(17)$ \\
2.500 & 0.0600 & 1.850 & 34.9 & $2.450(8)$ \\
3.000 & 0.0357 & 2.502 & 28.6 & $2.508(11)$ \\
3.448 & 0.0238 & 3.124 & 24.6 & $2.566(9)$ \\
4.000 & 0.0150 & 3.937 & 31.4 & $2.627(6)$ \\
5.000 & 0.0079 & 5.545 & 33.0 & $2.712(11)$ \\
6.000 & 0.0046 & 7.314 & 27.3 & $2.804(11)$ \\
8.000 & 0.0019 & 11.29 & 20.3 & $2.933(25)$ \\
\hline
\end{tabular}

\begin{tabular}{|c|c|c|c|c|}
\hline$R_{M} / R_{m}$ & $\varphi_{M}$ & $\Sigma_{A P}$ & $\frac{L_{\max }}{R_{M}}$ & $\rho_{3 c}^{\prime}$ \\
\hline \multicolumn{5}{|c|}{ 4-Ellipses } \\
\hline 2.000 & 0.1111 & 1.237 & 44.4 & $2.055(3)$ \\
\hline 2.500 & 0.0602 & 1.850 & 34.9 & $2.092(4)$ \\
\hline 3.000 & 0.0357 & 2.502 & 28.6 & $2.132(5)$ \\
\hline 3.448 & 0.0238 & 3.124 & 24.6 & $2.168(7)$ \\
\hline 4.000 & 0.0154 & 3.937 & 31.4 & $2.208(6)$ \\
\hline 6.000 & 0.0046 & 7.314 & 20.5 & $2.333(12)$ \\
\hline
\end{tabular}

TABLE VI. Data for bidisperse networks of disks or 4-ellipses, with bounding radii $R_{M}$ and $R_{m}$, and a fraction $\varphi_{M}=\varphi_{M, \max }=1 /\left(1+\tilde{R}^{3}\right)$ of large objects: polydispersity index $\Sigma_{A P}$, largest cell size $L_{\max }$ and percolation threshold $\rho_{3 c}^{\prime}$. 


\begin{tabular}{|c|c|c|c|c|c|c|}
\hline \multirow{2}{*}{\multicolumn{3}{|c|}{$a \quad \Sigma_{A P} \frac{L_{\max }}{R_{M}} \quad \rho_{3 c}^{\prime}$}} & & & & \multirow[b]{2}{*}{$\Sigma_{A P} \frac{L_{\max }}{R_{1}}$} \\
\hline & & & a $\quad \Sigma_{A P}$ & $\frac{L_{\max }}{R_{M}}$ & $\rho_{3 c}^{\prime}$ & \\
\hline \multicolumn{3}{|c|}{ Disks, $R_{M} / R_{m}=4$} & \multicolumn{3}{|c|}{4 -Ellipses, $R_{M} / R_{m}=4$} & Hexagons, $R_{M} / R_{m}=4$ \\
\hline 0.0 & 0.85340 & $2.341(6)$ & 0.00 .853 & 40 & $2.021(2)$ & $1.51 .19240 \quad 2.402(5)$ \\
\hline 0.5 & $0.956 \quad 40$ & $2.344(6)$ & 1.01 .070 & 20 & $2.034(4)$ & 2.01 .31540 \\
\hline 1.0 & $1.070 \quad 40$ & $2.362(6)$ & 1.51 .192 & 20 & $2.038(6)$ & $2.51 .42940 \quad 2.431(6)$ \\
\hline 1.5 & 1.192120 & $2.372(6)$ & 2.01 .315 & 20 & $2.043(5)$ & $4.01 .60820^{(=)} 2.458(20)$ \\
\hline 2.0 & $1.315 \quad 40$ & $2.383(6)$ & 2.51 .429 & $10^{(=)}$ & $2.061(20)$ & Hexagons, $R_{M} / R_{m}=8$ \\
\hline 2.5 & 1.429120 & $2.399(4)$ & 3.51 .586 & $10^{(=)}$ & $2.071(20)$ & 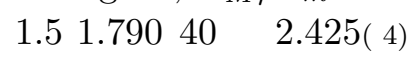 \\
\hline 3.0 & $1.52320^{(=)}$ & $2.412(20)$ & 4.01 .608 & 20 & $2.070(4)$ & $2.02 .18740 \quad 2.460$ (6) \\
\hline 3.5 & $1.58620^{(=)}$ & $2.421(20)$ & 4.51 .586 & $10^{(=)}$ & $2.074(20)$ & $2.52 .62620^{(=)} 2.499(20)$ \\
\hline 4.0 & 1.60820 & $2.411(15)$ & \multicolumn{3}{|c|}{ 4-Ellipses, $R_{M} / R_{m}=8$} & \\
\hline 5.0 & $1.52320^{(=)}$ & $2.414(20)$ & 0.01 .001 & 40 & $2.024(3)$ & Squares, $R_{M} / R_{m}=4$ \\
\hline 10.0 & $0.559 \quad 40$ & $2.323(2)$ & 1.01 .461 & 20 & $2.038(5)$ & $1.51 .19240 \quad 2.411(4)$ \\
\hline \multicolumn{3}{|c|}{ Disks, $R_{M} / R_{m}=8$} & 1.51 .790 & 20 & $2.050(5)$ & $2.51 .42940 \quad 2.441(5)$ \\
\hline 0.0 & 1.00140 & $2.343(5)$ & 2.02 .187 & 20 & $2.066(5)$ & $4.01 .60820^{(=)} 2.462(20)$ \\
\hline 0.5 & $1.200 \quad 40$ & $2.353(7)$ & 2.52 .626 & $10^{(=)}$ & $2.095(20)$ & Squares, $R_{M} / R_{m}=8$ \\
\hline 1.0 & 1.46140 & $2.371(6)$ & 3.53 .362 & $10^{(=)}$ & $2.141(20)$ & $1.51 .79020^{(=)} 2.437(20)$ \\
\hline 1.5 & $1.790 \quad 40$ & 2.391( 7$)$ & 4.03 .479 & $10^{(=)}$ & $2.159(20)$ & $2.52 .62620^{(=)} 2.505(20)$ \\
\hline 2.0 & $2.187 \quad 40$ & $2.420(7)$ & 4.53 .361 & $10^{(=)}$ & $2.153(20)$ & $4.03 .47920^{(=)} 2.596(20)$ \\
\hline 2.5 & 2.62640 & $2.451(8)$ & & & & \\
\hline 3.0 & $3.04820^{(=)}$ & $2.494(20)$ & & & & Triangles, $R_{M} / R_{m}=4$ \\
\hline 3.5 & $3.36220^{(=)}$ & $2.536(20)$ & & & & $1.51 .19220^{(=)} 2.341(20)$ \\
\hline 4.0 & 3.47920 & $2.549(11)$ & & & & $2.51 .42920^{(=)} 2.363(20)$ \\
\hline 5.0 & $3.04620^{(=)}$ & $2.505(20)$ & & & & $4.01 .60820^{(=)} 2.385(20)$ \\
\hline 10.0 & $0.57520^{(=)}$ & $2.324(20)$ & \multicolumn{3}{|c|}{ 4-Rectangles, $R_{M} / R_{m}=8$} & \multirow{4}{*}{ 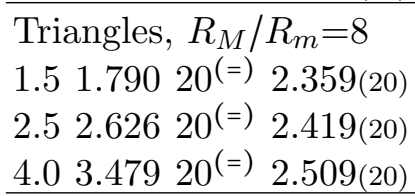 } \\
\hline \multicolumn{3}{|c|}{ Disks, $R_{M} / R_{m}=16$} & 4.03 .479 & $10^{(=)}$ & $2.173(20)$ & \\
\hline 0.0 & $1.070 \quad 40$ & $2.346(7)$ & \multicolumn{3}{|c|}{ 4-Lozenges:, $R_{M} / R_{m}=8$} & \\
\hline 0.5 & $1.352 \quad 40$ & $2.356(8)$ & 4.03 .479 & $10^{(=)}$ & $2.073(20)$ & \\
\hline 1.0 & 1.77940 & $2.375(6)$ & & & & \\
\hline 1.5 & $2.41620^{(=)}$ & $2.397(20)$ & & & & \\
\hline 2.0 & $3.33320^{(=)}$ & $2.440(20)$ & & & & \\
\hline 2.5 & $4.54920^{(=)}$ & $2.499(20)$ & & & & \\
\hline 4.0 & $7.62710^{(=)}$ & $2.698(20)$ & & & & \\
\hline
\end{tabular}

TABLE VII. Data for fracture networks of with power law size distribution in the range of bounding radii $R_{m}$ to $R_{M}$ : exponent $a$, polydispersity index $\Sigma_{A P}$, largest cell size $L_{\text {max }}$ and percolation threshold $\rho_{3 c}^{\prime}$. 


\begin{tabular}{|c|c|c|c|c|c|c|c|c|c|c|c|}
\hline$\tilde{R}$ & $\Sigma_{A P}$ & $\frac{L_{\max }}{R_{M}}$ & $\rho_{3 c}^{\prime}$ & $\tilde{R}$ & $\Sigma_{A P}$ & $\frac{L_{\max }}{R_{M}}$ & $\rho_{3 c}^{\prime}$ & $\tilde{R}$ & $\Sigma_{A P}$ & $\frac{L_{\max }}{R_{M}}$ & $\rho_{3 c}^{\prime}$ \\
\hline \multicolumn{4}{|c|}{ Disks, $a=0.0$} & \multicolumn{4}{|c|}{ Disks, $a=3.5$} & \multicolumn{4}{|c|}{ Hexagons, $a=1.5$} \\
\hline 4 & 0.853 & 40 & $2.341(6)$ & 4 & 1.586 & $20^{(=)}$ & $2.421(20)$ & 4 & 1.192 & 40 & $2.402(5)$ \\
\hline 8 & 1.001 & 40 & $2.343(5)$ & 8 & 3.362 & $20^{(=)}$ & $2.536(20)$ & 8 & 1.790 & 40 & $2.425(4)$ \\
\hline 16 & 1.070 & 40 & $2.346(7)$ & \multicolumn{4}{|c|}{ Disks, $a=4.0$} & \multicolumn{4}{|c|}{ Hexagons, $a=2.0$} \\
\hline \multicolumn{4}{|c|}{ Disks, $a=0.5$} & 2 & 0.645 & 40 & $2.331(4)$ & 4 & 1.315 & 40 & $2.418(4)$ \\
\hline 4 & 0.956 & 40 & $2.344(6)$ & 3 & 1.142 & 20 & $2.374(5)$ & 8 & 2.187 & 40 & $2.460(6)$ \\
\hline 8 & 1.200 & 40 & $2.353(7)$ & 4 & 1.608 & 20 & $2.41115)$ & \multicolumn{4}{|c|}{ Hexagons, $a=2.5$} \\
\hline 16 & 1.352 & 40 & $2.356(8)$ & 5 & 2.068 & 20 & $2.456(6)$ & 4 & 1.429 & 40 & $2.431(6)$ \\
\hline \multicolumn{4}{|c|}{ Disks, $a=1.0$} & 6 & 2.531 & $10^{(=)}$ & $2.496(20)$ & 8 & 2.626 & $20^{(=)}$ & $2.499(20)$ \\
\hline 4 & 1.070 & 40 & $2.362(6)$ & 8 & 3.479 & 20 & $2.549(11)$ & \multicolumn{4}{|c|}{ Squares, $a=1.5$} \\
\hline 8 & 1.461 & 40 & $2.371(6)$ & 10 & 4.463 & $10^{(=)}$ & $2.599(20)$ & 4 & 1.192 & 40 & $2.411(4)$ \\
\hline 16 & 1.779 & 40 & $2.375(6)$ & 12 & 5.485 & $10^{(=)}$ & $2.641(20)$ & 8 & 1.790 & $20^{(=)}$ & $37(20)$ \\
\hline \multicolumn{4}{|c|}{ Disks, $a=1.5$} & 16 & 7.627 & $10^{(=)}$ & $2.698(20)$ & \multicolumn{4}{|c|}{ Squares, $a=2.5$} \\
\hline 4 & 1.192 & 120 & $2.372(6)$ & \multicolumn{4}{|c|}{ Disks, $a=5.0$} & 4 & 1.429 & & $2.441(5)$ \\
\hline 8 & 1.790 & 40 & 2.391( 7$)$ & 4 & 1.523 & $20^{(=)}$ & $2.414(20)$ & 8 & 2.626 & $20^{(=)}$ & $2.505(20)$ \\
\hline 16 & 2.416 & $20^{(=)}$ & $2.397(20)$ & 8 & 3.046 & $20^{(=)}$ & $2.505(20)$ & \multicolumn{4}{|c|}{ Squares, $a=4.0$} \\
\hline \multicolumn{8}{|c|}{ Disks, $a=2.0$} & 4 & 1.608 & $20^{(=)}$ & $2.462(20)$ \\
\hline 4 & 1.315 & 40 & $2.383(6)$ & \multicolumn{4}{|c|}{ 4-Ellipses, $a=4.0$} & 8 & 3.479 & $20^{(=)}$ & $2.596(20)$ \\
\hline 8 & 2.187 & 40 & $2.420(7)$ & 2 & 0.645 & 40 & $2.016(3)$ & \multicolumn{4}{|c|}{ Triangles, $a=1.5$} \\
\hline 16 & 3.333 & $20^{(=)}$ & $2.440(20)$ & 3 & 1.142 & 20 & $2.041(4)$ & 4 & 1.192 & $20^{(=)}$ & $2.341(20)$ \\
\hline \multicolumn{4}{|c|}{ Disks, $a=2.5$} & 4 & 1.608 & 20 & $2.070(4)$ & 8 & 1.790 & $20^{(=)}$ & $2.359(20)$ \\
\hline 4 & 1.429 & 120 & $2.399(4)$ & 5 & 2.068 & 20 & $2.097(5)$ & \multicolumn{4}{|c|}{ Triangles, $a=2.5$} \\
\hline 8 & 2.626 & 40 & $2.451(8)$ & 6 & 2.531 & $10^{(=)}$ & $2.119(20)$ & 4 & 2.626 & $20^{(=)}$ & $2.419(20)$ \\
\hline 16 & 4.549 & $20^{(=)}$ & $2.499(20)$ & 8 & 3.479 & $10^{(=)}$ & $2.159(20)$ & 8 & 1.429 & $20^{(=)}$ & $2.363(20)$ \\
\hline \multicolumn{4}{|c|}{ Disks, $a=3.0$} & 10 & 4.463 & $10^{(=)}$ & $2.187(20)$ & \multicolumn{4}{|c|}{ Triangles, $a=4.0$} \\
\hline 4 & 1.523 & $20^{(=)}$ & $2.412(20)$ & 12 & 5.485 & $10^{(=)}$ & $2.214(20)$ & 4 & 1.608 & $20^{(=)}$ & $2.385(20)$ \\
\hline 8 & 3.048 & $20^{(=)}$ & $2.494(20)$ & 16 & 7.627 & $10^{(=)}$ & $2.260(20)$ & 8 & 3.479 & $20^{(=)}$ & $2.509(20)$ \\
\hline
\end{tabular}

TABLE VIII. Data for fracture networks of with power law size distribution with exponent $a$ : range of bounding radii $\tilde{R}=R_{M} / R_{m}$, polydispersity index $\Sigma_{A P}$, largest cell size $L_{\text {max }}$ and percolation threshold $\rho_{3 c}^{\prime}$. 


\section{RANDOM QUADRILATERALS}

This model is intended to introduce a class of random fracture networks reminiscent of real geological ones. Although it does not result from the modelling of field data, the fractures can take a great variety of shapes and sizes, which is more realistic than one or a few sets of regularly shaped objects. In addition, it provides a very demanding test case to assess whether results obtained in simple model situations apply in a more general setting.

The fractures are generated by randomly injecting four points in a disk of constant radius $R$ (see Fig.1). The cases where three points form a triangle that contains the fourth one are rejected. This occurs with a probability $35 /\left(12 \pi^{2}\right) \approx 0.296^{4}$. The remaining quadrilaterals have strongly polydisperse sizes and shapes that range from roughly square or roughly triangular to very elongated.

Their statistical geometric characteristics have been measured from a set of $10^{8}$ random realizations. The means, extremal values and standard deviations of the main ones for our purposes, namely the area $A$, the perimeter $P$, their product $A P$ and the shape factor $\tilde{\eta}_{g}$ (averaged according to Eq. 11) are given in Table IX. The maximal values of $A, P$ and $A P$ are reached for the square inscribed in the circle, with $A_{\diamond}=2 R^{2}, P_{\diamond}=4 \sqrt{2} R$ and $(A P)_{\diamond}=8 \sqrt{2} R^{3}$. The minimal value $1-\sqrt{3 / \pi}$ of $\tilde{\eta}_{g}$ also corresponds to this square.

The probability distributions of these parameters are shown in Fig.1. It appears that many quadrilaterals are very elongated. The shape factor $\tilde{\eta}_{g}$ exceeds 0.2 (which roughly corresponds to the value for 3-ellipses) with probability 0.5 and it exceeds 0.46 (which roughly corresponds to the value for 6 -ellipses) with probability 0.1 . The measures $A, P$ and $A P$ of the object size are also very widely spread.

\begin{tabular}{l|cccc}
\hline & Minimum & Average & Maximum & $\begin{array}{c}\text { Standard } \\
\text { deviation }\end{array}$ \\
\hline$A / R^{2}$ & 0 & 0.4895 & 2 & 0.2834 \\
$P / R$ & 0 & 3.2847 & $4 \sqrt{2} \approx 5.657$ & 0.7323 \\
$A P / R^{3}$ & 0 & 1.7860 & $8 \sqrt{2} \approx 11.314$ & 1.3457 \\
$\tilde{\eta}_{g}$ & $1-\sqrt{3 / \pi} \approx 0.0228$ & 0.2108 & 1 & \\
\hline
\end{tabular}

TABLE IX. Means, extrema and standard deviations of the area $A$, perimeter $P$, product $A P$ and shape factor $\tilde{\eta}_{g}$ of the random quadrilaterals.

\footnotetext{
${ }^{4}$ Sylvester J.J., On a Special Class of Questions on the Theory of Probabilities, Birmingham British Association Report, 39, 8-9 (1865).
} 


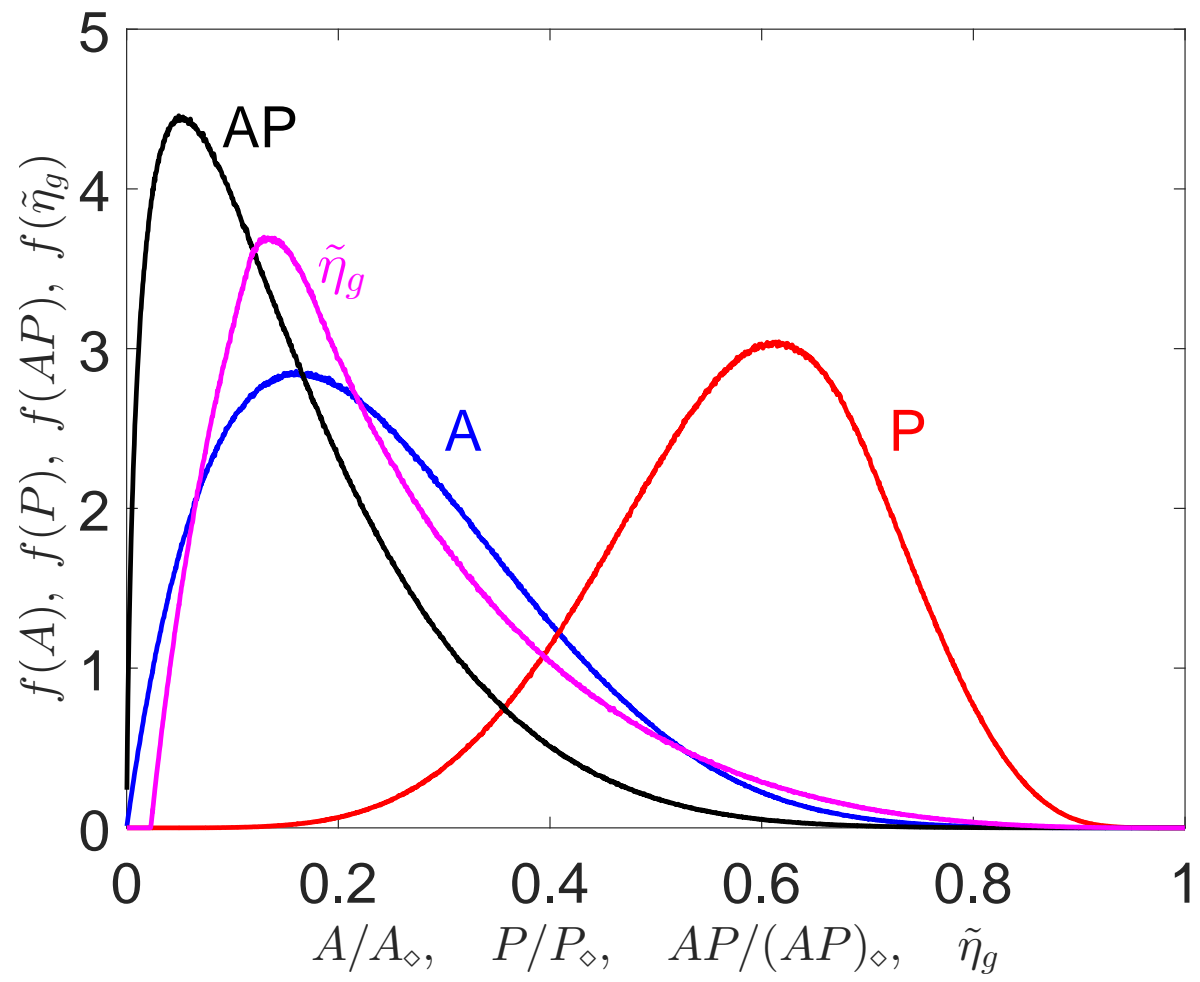

FIG. 1. Probability distribution functions of the area $A$, perimeter $P$, product $A P$ and shape factor $\tilde{\eta}_{g}$ of the random quadrilaterals. 


\section{MOMENTS OF THE CENTER-TO-CENTER DISTANCE OF INTERSECTING OBJECTS}

\begin{tabular}{ccc}
\hline \multicolumn{3}{l}{ dentical objects } \\
\hline \multicolumn{3}{c}{ n-Sided regular } \\
$n$ & $l^{(1)}$ & $l^{(2)}$ \\
3 & 0.8166 & 0.7500 \\
4 & 0.9477 & 0.9999 \\
6 & 1.0597 & 1.2501 \\
20 & 1.1500 & 1.4755 \\
\hline
\end{tabular}

Ellipses with aspect ratio $f$

$\begin{array}{ccc}f & l^{(1)} & l^{(2)} \\ 1 & 1.1593 & 1.5000 \\ 1.5 & 0.9693 & 1.0559 \\ 2 & 0.8785 & 0.8776 \\ 3 & 0.7950 & 0.7323 \\ 4 & 0.7583 & 0.6746 \\ 6 & 0.7274 & 0.6281 \\ 8 & 0.7150 & 0.6102 \\ 16 & 0.7014 & 0.5910\end{array}$

Rectangles with aspect ratio $f$ f $l^{(1)} \quad l^{(2)}$

$\begin{array}{lll}1 & 0.9477 & 0.9999\end{array}$

$2 \quad 0.9075 \quad 0.9333$

$\begin{array}{lll}4 & 0.8403 & 0.8237\end{array}$

$\begin{array}{lll}6 & 0.8105 & 0.7749\end{array}$

$\begin{array}{lll}8 & 0.7950 \quad 0.7489\end{array}$

$\begin{array}{lll}16 & 0.7711 \quad 0.7085\end{array}$

Losanges with aspect ratio $f$

$f l^{(1)} \quad l^{(2)}$

$\begin{array}{lll}1 & 0.9477 & 0.9999\end{array}$

$\begin{array}{lll}2 & 0.7402 & 0.6250\end{array}$

$\begin{array}{lll}3 & 0.6897 & 0.5556\end{array}$

$4 \quad 0.6695 \quad 0.5312$

$8 \quad 0.6478 \quad 0.5078$

\begin{tabular}{ccc}
\hline Unit disk + disk with radius $r$ & \\
$r$ & $l^{(1)}$ & $l^{(2)}$ \\
1 & 1.1593 & 1.5000 \\
$2 / 3$ & 0.9722 & 1.0555 \\
$1 / 2$ & 0.8847 & 0.8750 \\
$2 / 5$ & 0.8349 & 0.7800 \\
$1 / 3$ & 0.8031 & 0.7222 \\
0.3112 & 0.7927 & 0.7040 \\
0.2900 & 0.7830 & 0.6871 \\
$1 / 4$ & 0.7650 & 0.6562 \\
$1 / 5$ & 0.7433 & 0.6200 \\
$1 / 6$ & 0.7293 & 0.5972 \\
$1 / 8$ & 0.7124 & 0.5703 \\
$f$ & $l^{(1)}$ & $l^{(2)}$ \\
4 & 0.9428 & 1.0043 \\
8 & 0.9011 & 0.9203
\end{tabular}

Unit disk + ellipse with aspect ratio $f$, same area

\begin{tabular}{ccc}
$f$ & $l^{(1)}$ & $l^{(2)}$ \\
4 & 1.3459 & 2.1041 \\
8 & 1.6374 & 3.2376 \\
\hline Unit $n$-sided polygon + same shape, smaller size $(1 / 4)$ \\
$n$ & $l^{(1)}$ & $l^{(2)}$ \\
4 & 0.5315 & 0.3281 \\
6 & 0.6224 & 0.4375 \\
20 & 0.6982 & 0.5469 \\
Unit $n_{1}$-sided regular polygon $+n_{2}$-sided \\
$n_{1}, n_{2}$ \\
20,4 (same bounding radius) & 1.0485 & 0.6456 \\
20,4 (same area) & 1.1645 & 1.2246 \\
6,3 (same bounding radius) & 0.9363 & 0.9779 \\
\hline
\end{tabular}

TABLE X. Moments $l^{(1)}$ and $l^{(2)}$ of the center-to-center distance of intersecting objects with identical (left) or different (right) shapes and/or sizes, determined by Monte Carlo numerical integration. The objects have a unit bounding radius, unless otherwise stated. 